Journal of Neuroscience Methods, in press (2008).

\title{
Characterizing synaptic conductance fluctuations in cortical neurons and their influence on spike generation
}

\author{
Zuzanna Piwkowska $^{1}$, Martin Pospischil ${ }^{1}$, Romain Brette ${ }^{2}$, Julia Sliwa ${ }^{1,3}$, \\ Michelle Rudolph-Lilith ${ }^{1}$, Thierry Bal ${ }^{1}$ and Alain Destexhe ${ }^{1, *}$ \\ 1: Unité de Neurosciences Intégratives et Computationnelles (UNIC), \\ CNRS, 91198 Gif-sur-Yvette, France \\ 2: Équipe Odyssée (ENS/INRIA/ENPC), École Normale Supérieure, Paris, France \\ 3: Present address: Centre de Neuroscience Cognitive (CNC), UMR 5229, CNRS, 69675 Bron, France \\ *: corresponding author at address 1 above; \\ Tel: 33-1-6982-3435, Fax: 33-1-6982-3427; destexhe@unic.cnrs-gif.fr
}

\begin{abstract}
Abbreviated title: Characterizing synaptic conductance fluctuations
Keywords: Intracellular recordings; Conductance analysis; Cerebral cortex; Dynamic-clamp; Computational models; Spike-triggered average; Inhibition
\end{abstract}

\begin{abstract}
Cortical neurons are subject to sustained and irregular synaptic activity which causes important fluctuations of the membrane potential $\left(\mathrm{V}_{m}\right)$. We review here different methods to characterize this activity and its impact on spike generation. The simplified, fluctuating point-conductance model of synaptic activity provides the starting point of a variety of methods for the analysis of intracellular $\mathrm{V}_{m}$ recordings. In this model, the synaptic excitatory and inhibitory conductances are described by Gaussian-distributed stochastic variables, or "colored conductance noise". The matching of experimentally recorded $\mathrm{V}_{m}$ distributions to an invertible theoretical expression derived from the model allows the extraction of parameters characterizing the synaptic conductance distributions. This analysis can be complemented by the matching of experimental $\mathrm{V}_{m}$ power spectral densities (PSDs) to a theoretical template, even though the unexpected scaling properties of experimental PSDs limit the precision of this latter approach. Building on this stochastic characterization of synaptic activity, we also propose methods to qualitatively and quantitatively evaluate spike-triggered averages of synaptic time-courses preceding spikes. This analysis points to an essential role for synaptic conductance variance in determining spike times. The presented methods are evaluated using controlled conductance injection in cortical neurons in vitro with the dynamic-clamp technique. We review their applications to the analysis of in vivo intracellular recordings in cat association cortex, which suggest a predominant role for inhibition in determining both sub- and supra-threshold dynamics of cortical neurons embedded in active networks.
\end{abstract}




\section{Introduction}

Cerebral cortical networks can generate states of intense and irregular activity, which are characterized by low-amplitude "desynchronized" fast activity in the electroencephalogram (EEG), a defining feature of the awake state. Intracellular measurements in awake animals (Woody and Gruen, 1978; Matsumura et al., 1988; Baranyi et al., 1993; Steriade et al., 2001; Timofeev et al., 2001; Rudolph et al., 2007) have shown that cortical neurons are depolarized (about $-60 \mathrm{mV}$ ), have a low input resistance, their membrane potential $\left(\mathrm{V}_{m}\right)$ fluctuates, and they fire irregularly and sustainedly. During slow-wave sleep, or under several types of anesthetics (such as urethane or ketamine-xylazine), the $\mathrm{V}_{m}$ displays "up-" (depolarized) and "down-" (hyperpolarized) states, which are paralleled with EEG slow waves (Metherate and Ashe, 1993; Steriade et al., 1993; Steriade et al., 2001; Timofeev et al., 2001). During the up-state, the EEG is desynchronized and the $\mathrm{V}_{m}$ of cortical neurons is depolarized and highly fluctuating, similar to the sustained activity found in awake animals (Destexhe et al., 2007). Up- and down-states have also been found in ferret cortical slices using high-potassium and low-calcium extracellular media (Sanchez-Vives and McCormick, 2000) and in rat entorhinal slices as a function of the metabolic state (Cunningham et al., 2006): these experiments indicate that intracortical circuits are able to generate such states, presumably through recurrent excitation and inhibition. TTX block of action potentials in vivo (Paré et al., 1998) and CNQX block of excitatory synapses in vitro (Cunningham et al., 2006) abolish the depolarized, fluctuating states, which confirms their synaptic origin.

The recurrent activity of cortical networks has been investigated using computational models at different levels and including various degrees of biological detail. Large networks of formal (or simple spiking) neurons allow the analytic derivation (or numerical confirmation) of conditions for different classes of network activity, such as oscillations or deterministic chaos (Van Vreeswijk and Sompolinsky, 1996; Roxin et al., 2005; Barak and Tsodyks, 2007). Network models incorporating a realistic diversity of cell types and details of cortical connectivity allow quantitative predictions to be obtained through numerical simulations (Compte et al., 2003; Hill and Tononi, 2005). At the single neuron level, detailed models can include the cell's morphology, a variety of intrinsic ion channels and distributed synaptic inputs: studies of such models assess the impact of massive input from the cortical network on dendritic processing, spike train statistics, neuronal responsiveness and integrative properties (Bernander et al., 1991; Destexhe and Paré, 1999; Rudolph and Destexhe 2003b,c; Destexhe et al., 2003b). However, the parameterization of all those models requires a large amount of information that cannot be obtained from any single experiment, which complicates the comparison of simulation results with biological data.

A complementary approach consists in developing and studying models that are relatively simple, but contain variables and parameters that can be related to quantities directly measured in experiments. Various mathematical studies of the firing dynamics of neurons with conductance-based synaptic inputs have been proposed (see for example Burkitt et al., 2003; Moreno-Bote and Parga, 2005; Muller et al., 2007). Other authors investigated the integration of multiple current- or conductance-based inputs driven by Poisson spike trains, in terms of $\mathrm{V}_{m}$ fluctuations and output firing rate of simple point model neurons (Kuhn et al., 2004). In the approach proposed by Destexhe and colleagues (Destexhe et al., 2001), the effective impact at the soma of thousands of single synapses, respectively excitatory and inhibitory, activated by such Poisson spike trains, is represented by only two stochastic conductance variables, $g_{e}(t)$ and $g_{i}(t)$, modeled as Ornstein-Uhlenbeck (Brownian-motion-like) processes. This fluctuating point-conductance model describes the evolution of the subthreshold $\mathrm{V}_{m}$ of a single-compartment neuron by a passive membrane equation with the two additional stochastic conductance variables $g_{e}(t)$ and $g_{i}(t)$. We present here how this model can be used in close combination with electrophysiological experiments to investigate the properties and the impact of cortical recurrent activity at the single neuron level. 
More specifically, we critically review, on the basis of new as well as already published data, applications of the point-conductance model of synaptic activity to the analysis of intracellular recordings in cortical neurons: in each case, we first briefly describe the method of analysis, then we show how it can be validated experimentally by using controlled "conductance injection" in biological neurons with dynamic-clamp, and finally we present the application of the method to the analysis of real synaptic activity. We especially focus on recently developed approaches for determining which of the conductance configurations occurring in the fluctuating synaptic activity trigger spikes.

\section{Methods}

\subsection{Computational methods}

Computational models were based on single-compartment neurons described by the following membrane equation:

$$
C \frac{d V}{d t}=-G_{L}\left(V-E_{L}\right)-g_{e}\left(V-E_{e}\right)-g_{i}\left(V-E_{i}\right)+I_{e x t}
$$

where $C$ denotes the membrane capacitance, $I_{\text {ext }}$ a stimulation current, $G_{L}$ the leak conductance and $E_{L}$ the leak reversal potential. $g_{e}(t)$ and $g_{i}(t)$ are stochastic excitatory and inhibitory conductances, with respective reversal potentials $E_{e}$ and $E_{i}$.

These effective synaptic conductances were described by the following Ornstein-Uhlenbeck model (Destexhe et al., 2001):

$$
\begin{aligned}
\frac{d g_{e}(t)}{d t} & =-\frac{1}{\tau_{e}}\left[g_{e}(t)-g_{e 0}\right]+\sqrt{\frac{2 \sigma_{e}^{2}}{\tau_{e}}} \xi_{e}(t) \\
\frac{d g_{i}(t)}{d t} & =-\frac{1}{\tau_{i}}\left[g_{i}(t)-g_{i 0}\right]+\sqrt{\frac{2 \sigma_{i}^{2}}{\tau_{i}}} \xi_{i}(t),
\end{aligned}
$$

where $g_{e 0}$ and $\sigma_{e}^{2}$ are, respectively, the mean value and variance of the excitatory conductance, $\tau_{e}$ is the excitatory time constant, and $\xi_{e}(t)$ is a Gaussian white noise source with zero mean and unit standard deviation. The inhibitory conductance $g_{i}(t)$ is described by an equivalent equation (Eq. 3) with parameters $g_{i 0}, \sigma_{i}^{2}, \tau_{i}$ and noise source $\xi_{i}(t)$.

In some simulations, the voltage-dependent conductances responsible for action potentials, $g_{N a}$ and $g_{K d}$ (with respective reversals $E_{N a}$ and $E_{K}$ ), were included. They were described by Hodgkin-Huxley type models (with equations and parameters identical as described in Destexhe et al., 2001, and references therein), resulting in the following equation:

$$
C \frac{d V}{d t}=-G_{L}\left(V-E_{L}\right)-g_{N a}\left(V-E_{N a}\right)-g_{K d}\left(V-E_{K}\right)-g_{e}\left(V-E_{e}\right)-g_{i}\left(V-E_{i}\right)+I_{e x t} .
$$

Simulations were performed on LINUX workstations using the NEURON simulation environment (Hines and Carnevale, 1997). 


\subsection{Biological preparation}

In vitro experiments were performed on 380-400 $\mu$ m thick coronal or sagittal slices from the lateral portions of pentobarbital-anesthetized adult ferret (Marshall Europe, Lyon) and guinea-pig (CPA, Olivet, France) occipital cortex, as described previously (Rudolph et al., 2004; Pospischil et al., 2007). Slices were maintained in an interface style recording chamber at $33-35^{\circ} \mathrm{C}$ in slice solution containing (in $\mathrm{mM}$ ) $124 \mathrm{NaCl}$, $2.5 \mathrm{KCl}, 1.2 \mathrm{MgSO}_{4}, 1.25 \mathrm{NaHPO}_{4}, 2 \mathrm{CaCl}_{2}, 26 \mathrm{NaHCO}_{3}$, and 10 dextrose and aerated with 95\% $\mathrm{O}_{2}-$ $5 \% \mathrm{CO}_{2}$ to a final $\mathrm{pH}$ of 7.4. In some experiments on ferret cortical slices, after approximately 1 hour, the solution was modified to contain $1 \mathrm{mM} \mathrm{MgSO}_{4}, 1 \mathrm{mM} \mathrm{CaCl}_{2}$ and $3.5 \mathrm{mM} \mathrm{KCl}$ (Sanchez-Vives and McCormick, 2000) in order to obtain up-states. Intracellular recordings following two hours of recovery were performed in all cortical layers on electrophysiologically identified regular spiking and intrinsically bursting cells.

All research procedures concerning the experimental animals and their care adhered to the American Physiological Society's Guiding Principles in the Care and Use of Animals, to the European Council Directive 86/609/EEC and to European Treaties series no. 123, and was also approved by the local ethics committee "Ile-de-France Sud" (certificate no. 05-003).

We also review data from intracellular recordings in cat association cortex in vivo, which were described in detail elsewhere (Rudolph et al., 2005; Steriade et al., 2001; Rudolph et al., 2007).

\subsection{Electrophysiology}

Sharp electrodes for intracellular recordings were made on a Sutter Instruments P-87 micropipette puller from medium-walled glass (WPI, 1BF100) and beveled on a Sutter Instruments beveler (BV-10M). Micropipettes were filled with 1.2-2 $\mathrm{M}$ potassium acetate $-4 \mathrm{mM}$ potassium chloride and had resistances of 65-110 M $\Omega$ after beveling. An Axoclamp 2B amplifier (Axon Instruments) was used for $\mathrm{V}_{m}$ recording and current injection. A Digidata 1322A card (Axon Instruments) was used for data acquisition at $20 \mathrm{kHz}$.

The dynamic-clamp technique (Robinson et al., 1993; Sharp et al., 1993) was used to inject computergenerated conductances in real neurons. Dynamic-clamp experiments were run as described previously (Rudolph et al., 2004, Pospischil et al., 2007) using the hybrid RT-NEURON environment (developed by G. Le Masson, INSERM 358, Université Bordeaux 2), which is a modified version of NEURON (Hines and Carnevale, 1997) running in real-time under the Windows 2000 operating system (Microsoft Corp.). In these experiments, the injected current $I_{\text {DynClamp }}$ was determined from the fluctuating conductances $g_{e}(t)$ and $g_{i}(t)$ modeled with Ornstein-Uhlenbeck processes (Eqs. 2.35) as well as from the difference between the recorded membrane voltage $V$ and the respective reversal potentials:

$$
I_{\text {DynClamp }}=-g_{e}\left(V-E_{e}\right)-g_{i}\left(V-E_{i}\right) .
$$

The contamination of measured membrane voltage by electrode artefacts was avoided either through the use of the discontinuous current-clamp mode (in which current injection and voltage recording alternate at frequencies of $2-3 \mathrm{kHz}$ with our electrodes) or with Active Electrode Compensation, a novel, highresolution digital on-line compensation technique we have recently developed (Brette et al., 2005, 2007; Rudolph et al., 2005). 


\subsection{Data analysis}

The PC-based software ELPHY (developed by G. Sadoc, CNRS Gif-sur-Yvette, ANVAR and Biologic), Statview, Excel, custom-written C-code and Neuron-code were used for analyses. All values are given as average \pm standard deviation.

\subsubsection{VmD analysis}

In dynamic-clamp experiments re-creating up-states with conductance injection, two approaches were used. In 3 cells, conductance estimates were computed for different, realistic values of leak conductance and cell capacitance, and were then tested against the real up-states in dynamic-clamp. In 2 cells, leak conductance and cell capacitance were estimated from the response to short current pulses, and those values were then used for conductance estimation: the estimated conductance parameters, when used for dynamic-clamp injection, proved to allow successful matching of the real up-state in terms of $\mathrm{V}_{m}$ distributions. In all cases, conductance estimation was done during the recording using ELPHY.

VmD analysis of the in vivo data reviewed here is described in Rudolph et al., 2005 and Rudolph et al., 2007.

\subsubsection{Power Spectral Density (PSD) analysis}

Power spectra of $\mathrm{V}_{m}$ activity were fit to an analytic template (see Results) using a simplex fitting algorithm (Press et al., 1986). Different initial conditions ("first guesses") were given to the fitting procedure to ensure that there was no convergence to local minima. Some fits were realized by fitting both the amplitude of excitatory and inhibitory components $\left(A_{e}\right.$ and $A_{i}$; see Results), as well as the time constants $\left(\tau_{e}\right.$ and $\left.\tau_{i}\right)$. In other cases, it was not possible to fit 4 parameters from the experimental PSD. In such cases (typically from in vivo data), the fit was performed with a single amplitude component $\left(A_{e}=A_{i}\right)$. In all cases, the effective membrane time constant $\left(\tilde{\tau}_{m}\right)$ was fixed to the value estimated from the recordings.

\subsubsection{Spike-Triggered Average (STA) analysis}

For each conductance injection, spikes were detected using a threshold at $-30 \mathrm{mV}$. Inter-spike-intervals (ISIs) were computed and for further analysis, a stable region in terms of ISI distribution was used (as assessed by a non-significant Spearman correlation test between ISI duration and time), including $673 \pm$ 493 spikes. When investigating the impact of frequency on the accuracy of the estimates, the same number of spikes (52) was used for all analyzed injections. Spikes following ISIs of at least $100 \mathrm{~ms}$ were then selected. $50 \mathrm{~ms}$-long pieces of $\mathrm{V}_{m}, g_{e}$ and $g_{i}$ preceding each selected spike were averaged to obtain the "measured STAs". Using the recorded conductance traces, we checked that excluding spikes following ISIs shorter than $100 \mathrm{~ms}$ did not affect the measured STAs in an important way (the difference in conductance variation before the spike was of $-0.14 \pm 0.35 \mathrm{nS}$ for excitation, and of $0.5 \pm 0.7 \mathrm{nS}$ for inhibition). However, in order to compare with the STA extraction method based on the $\mathrm{V}_{m} \mathrm{STA}$, it is important to exclude short ISIs from the analysis to minimize contamination of the $\mathrm{V}_{m}$ STA by conductances related to preceding spikes.

For conductance STAs extraction based on the $\mathrm{V}_{m}$ STA, parameters were obtained in the following way: 
responses to depolarizing current pulses were used to estimate the membrane capacitance from the time constant of exponential fits to the decay of the $\mathrm{V}_{m}$, and the leak conductance was obtained by dividing the average voltage during conductance injection, relative to rest, by the average injected current. This results in an effective leak conductance possibly comprising different types of voltage-dependent conductances. An indication of the validity of this approach is provided by the estimated conductance STAs: the important variation of this effective leak conductance on the time scale of the length of the analysis window (for example due to conductances underlying spike frequency adaptation) would result in alterations of the stationary phase, which we did not observe when excluding ISIs shorter than $100 \mathrm{~ms}$. More details about the application of the STA analysis to in vivo data can be found in Rudolph et al. (2007).

To quantify both the measured and the extracted conductance STAs, we fitted the conductance time courses using the exponential template

$$
g_{e}(t)=g_{e 0}^{S T A}\left[1+k_{e} \exp \frac{\left(t-t_{0}\right)}{\tau_{g_{e}}^{S T A}}\right],
$$

for excitation, and an equivalent equation for inhibition. Here, $t_{0}$ stands for the time of the spike, $k_{e}$ quantifies the maximal increase/decrease of conductance prior to the spike $\left(\Delta g_{e}=g_{e 0} k_{e}\right)$, with time constant $\tau_{g_{e}}^{S T A}$, and $g_{e 0}^{S T A}$ is the average baseline conductance (see Results).

\section{Results}

The point-conductance model of recurrent cortical activity describes the evolution of the subthreshold $\mathrm{V}_{m}$ of a point neuron based on two effective fluctuating conductances $g_{e}$ and $g_{i}$ (Destexhe et al., 2001; see Methods for equations). The basic assumption of this model is that the synaptic conductances can be described as Gaussian-distributed stochastic variables. It was shown that the well-known Ornstein-Uhlenbeck model of Brownian noise (Uhlenbeck and Ornstein, 1930) approximates very well the total synaptic conductances resulting from a large number of simulated conductance-based synaptic inputs (Destexhe et al., 2001; Destexhe and Rudolph, 2004). The fitting of a Gaussian model to the total synaptic conductances seen at the soma, in simulations performed using realistic cortical neuron morphologies and distributed synaptic inputs, indicates the following correspondences between variables: the time constants $\left(\tau_{e}, \tau_{i}\right)$ are identical to the decay time constants of synaptic currents. The average conductance $\left(g_{e 0}, g_{i 0}\right)$ is related to the overall (integrated) conductance, which depends on the release frequency of the corresponding Poisson inputs, the quantal conductance and the decay time of synaptic currents. The variance of the conductances $\left(\sigma_{e}^{2}, \sigma_{i}^{2}\right)$ is related to the same parameters, as well as to the amount of correlation between inputs of the same type (Destexhe et al., 2001). Correlations between presynaptic spike trains seem necessary in order to account for the high amplitude of $\mathrm{V}_{m}$ fluctuations observed in vivo (e.g., Destexhe and Paré, 1999; Léger et al., 2005).

It has to be noted that the Gaussian model of synaptic conductances can only be considered as an approximation. As shown theoretically using the shot noise formalism, in the presence of very strong correlations, or a low mean presynaptic rate, this approximation might fail (Richardson and Gerstner, 2005; Rudolph and Destexhe, 2006; see also Kuhn et al., 2003). In this case, effective synaptic conductances might be better described by a gamma distribution. However, the Gaussian model seems appropriate, even in the presence of correlations, as long as the ratio of conductance standard-deviation to conductance mean remains small (so that the presence of negative conductances is negligible). Experimental support for this assumption has been obtained using voltage-clamp conductance measurements (Destexhe et al., 2003a). 
The theoretical and numerical analysis of the point-conductance model has led to various useful derivations: an invertible expression for the steady-state distribution of $\mathrm{V}_{m}$ fluctuations (Rudolph and Destexhe, 2003a, 2005), an expression for the power spectral density of $\mathrm{V}_{m}$ fluctuations (Destexhe and Rudolph, 2004), a geometrical analysis of the configuration of conductances directly preceding spikes, and a probabilistic method for calculating the most likely conductance time course preceding spikes given an average $\mathrm{V}_{m}$ time course (Pospischil et al., 2007). We now proceed to examine in more detail the applications of these computational results to the analysis of intracellular recordings in cortical neurons.

\subsection{The VmD method for extracting synaptic conductance parameters}

\subsubsection{Outline of the VmD method}

The model described by Eqs. 13 has been thoroughly studied theoretically and numerically. This model describes the subthreshold $\mathrm{V}_{m}$ fluctuations of a neuron subject to fluctuating conductances $g_{e}$ and $g_{i}$. Different analytic approximations have been proposed to describe the steady-state distribution of these $\mathrm{V}_{m}$ fluctuations (Rudolph and Destexhe, 2003a, 2005; Richardson, 2004; Lindner and Longtin, 2006; for a comparative study, see Rudolph and Destexhe, 2006). One of these expressions is invertible (Rudolph and Destexhe, 2003a, 2005), which enables one to directly estimate the parameters $\left(g_{e 0}, g_{e 0}, \sigma_{e}, \sigma_{i}\right)$ from experimentally calculated $\mathrm{V}_{m}$ distributions. This constitutes the basis of the $\mathrm{VmD}$ method (Rudolph et al., 2004), which we outline below.

The essential idea behind the $\mathrm{VmD}$ method is to fit an analytic expression to the steady-state subthreshold $\mathrm{V}_{m}$ distribution obtained experimentally, and yield estimates of the parameters (mean, variance) of the underlying synaptic conductances. Among the different analytic expressions outlined above, we consider the following Gaussian approximation of the steady-state $\mathrm{V}_{m}$ distribution:

$$
\rho(V) \sim \exp \left[-\frac{(V-\bar{V})^{2}}{2 \sigma_{V}^{2}}\right]
$$

where $\bar{V}$ is the average $\mathrm{V}_{m}$ and $\sigma_{V}$ its standard deviation. This expression provides an excellent approximation of the $\mathrm{V}_{m}$ distributions obtained from models and experiments (Rudolph et al., 2004), because the $\mathrm{V}_{m}$ distributions obtained experimentally show little asymmetry (for up-states and activated states; for specific examples, see Figs. 1 and 2, and Rudolph et al., 2004, 2005, 2007).

One main advantage of this Gaussian approximation is that it can be inverted, which leads to expressions of the synaptic noise parameters as a function of the $\mathrm{V}_{m}$ measurements, $\bar{V}$ and $\sigma_{V}$. By fixing the values of $\tau_{e}$ and $\tau_{i}$, which are related to the decay time of synaptic currents and can be estimated from voltage-clamp data and/or current-clamp by using power spectral analysis (see Section 3.2), we remain with four parameters to estimate: the means $\left(g_{e}, g_{i 0}\right)$ and standard deviations $\left(\sigma_{e}, \sigma_{i}\right)$ of excitatory and inhibitory synaptic conductances. To extract these four conductance parameters from the membrane probability distribution, Eq. 7 is, however, insufficient because it is characterized by only two parameters $\left(\bar{V}, \sigma_{V}\right)$. To solve this problem, one possibility is to consider two $\mathrm{V}_{m}$ distributions obtained at two different constant levels of in-

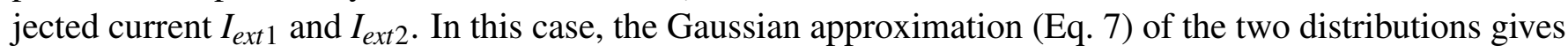
two mean $\mathrm{V}_{m}$ values, $\bar{V}_{1}$ and $\bar{V}_{2}$, and two standard deviation values, $\sigma_{V 1}$ and $\sigma_{V 2}$. The resulting system of four equations relating $\mathrm{V}_{m}$ parameters with conductance parameters can now be solved for four unknowns: 


$$
\begin{aligned}
& g_{\{e, i\} 0}= \frac{\left(I_{e x t 1}-I_{e x t 2}\right)\left[\sigma_{V 2}^{2}\left(E_{\{i, e\}}-\bar{V}_{1}\right)^{2}-\sigma_{V 1}^{2}\left(E_{\{i, e\}}-\bar{V}_{2}\right)^{2}\right]}{\left[\left(E_{e}-\bar{V}_{1}\right)\left(E_{i}-\bar{V}_{2}\right)+\left(E_{e}-\bar{V}_{2}\right)\left(E_{i}-\bar{V}_{1}\right)\right]\left(E_{\{e, i\}}-E_{\{i, e\}}\right)\left(\bar{V}_{1}-\bar{V}_{2}\right)^{2}} \\
&-\frac{\left(I_{e x t 1}-I_{e x t 2}\right)\left(E_{\{i, e\}}-\bar{V}_{2}\right)+\left[I_{e x t 2}-G_{L}\left(E_{\{i, e\}}-E_{L}\right)\right]\left(\bar{V}_{1}-\bar{V}_{2}\right)}{\left(E_{\{e, i\}}-E_{\{i, e\}}\right)\left(\bar{V}_{1}-\bar{V}_{2}\right)}, \\
& \sigma_{\{e, i\}}^{2}=\frac{2 C\left(I_{e x t 1}-I_{e x t 2}\right)\left[\sigma_{V 1}^{2}\left(E_{\{i, e\}}-\bar{V}_{2}\right)^{2}-\sigma_{V 2}^{2}\left(E_{\{i, e\}}-\bar{V}_{1}\right)^{2}\right]}{\tilde{\tau}_{\{e, i\}}\left[\left(E_{e}-\bar{V}_{1}\right)\left(E_{i}-\bar{V}_{2}\right)+\left(E_{e}-\bar{V}_{2}\right)\left(E_{i}-\bar{V}_{1}\right)\right]\left(E_{\{e, i\}}-E_{\{i, e\}}\right)\left(\bar{V}_{1}-\bar{V}_{2}\right)^{2}} .
\end{aligned}
$$

Here, $\tilde{\tau}_{\{e, i\}}$ are effective time constants given by (Rudolph and Destexhe, 2005):

$$
\tilde{\tau}_{\{e, i\}}=\frac{2 \tau_{\{e, i\}} \tilde{\tau}_{m}}{\tau_{\{e, i\}}+\tilde{\tau}_{m}},
$$

where $\tilde{\tau}_{m}=C /\left(G_{L}+g_{e 0}+g_{i 0}\right)$ is the effective membrane time constant.

These relations enable us to estimate global characteristics of network activity, such as mean excitatory $\left(g_{e 0}\right)$ and inhibitory $\left(g_{i 0}\right)$ synaptic conductances, as well as their respective variances $\left(\sigma_{e}^{2}, \sigma_{i}^{2}\right)$, from the sole knowledge of the $\mathrm{V}_{m}$ distributions obtained at two different levels of injected current. This $\mathrm{VmD}$ method was tested using computational models and dynamic-clamp experiments (Rudolph et al., 2004) and was also used to extract conductances from different experimental conditions in vivo (Rudolph et al., 2005, 2007; Zou et al., 2005).

\subsubsection{Testing the VmD method with dynamic-clamp}

Taking advantage of the possibility, given by the dynamic-clamp technique (see Methods), to mimic in a finely controlled way the fluctuating conductances $g_{e}$ and $g_{i}$ in biological neurons, we performed in vitro tests of the VmD method (Rudolph et al., 2004; Piwkowska et al., 2004). In a first test (in 5 neurons), we computed $\mathrm{V}_{m}$ distributions selectively during periods of subthreshold activity collected within up-states recorded in ferret cortical slices, we subsequently extracted conductance parameters from Gaussian fits to these distributions, and finally we used the estimated parameters to inject fluctuating conductances in dynamic-clamp in the same cell, during down-states. Fig $1 \mathrm{C}$ shows a typical example of a real up-state and, shortly after, an up-state re-created in dynamic-clamp. We confirmed that the $\mathrm{V}_{m}$ distributions are very similar in the two cases (see Rudolph et al., 2004 for more details). This test shows that the $\mathrm{V}_{m}$ distributions observed experimentally in vitro during recurrent cortical activity can be accounted for by the proposed point-conductance model. We also re-estimated known parameters of synaptic conductances $\left(\mathrm{g}_{e 0}, g_{i 0}, \sigma_{e}, \sigma_{i}\right)$ injected in dynamic-clamp from the resulting $\mathrm{V}_{m}$ distributions: the match between actual and estimated values is shown in Fig. 1 $1 \mathrm{~B}$. This second test indicates that the passive approximation for the membrane behavior holds in the studied case. In these tests, we did not consider the issue of the estimation of $\tau_{e}$ and $\tau_{i}$ and assumed these values are known.

\subsubsection{Analysis of intracellular recordings of cortical neurons in vivo}

The VmD method was then applied to analyze intracellular recordings in anesthetized (Rudolph et al., 2005), as well as naturally sleeping and awake cats (Rudolph et al., 2007). 
In the first study, recordings were performed in cat association cortex under ketamine-xylazine anesthesia, during the slow oscillation typical of this anesthetic and resembling slow-wave-sleep, as well as during prolonged periods of activity, triggered by brain stem (PPT) stimulation, and with activity similar to that of the aroused brain. The $\mathrm{VmD}$ method was used to extract synaptic conductance parameters underlying the $\mathrm{V}_{m}$ fluctuations of the up-states of the slow oscillation, as well as those underlying the continuous fluctuations following PPT stimulation. In both cases, the average estimated inhibitory conductance $g_{i 0}$ was markedly higher than the average estimated excitatory conductance $g_{e 0}$, and similarly the estimated variance of inhibition $\sigma_{i}^{2}$ was higher than the variance of excitation $\sigma_{e}^{2}$.

The second study shows similar results across the natural wake-sleep cycle of the cat (Fig. 2): for a majority of cells, especially during slow-wave-sleep up-states, inhibition dominated in terms of both mean and variance. At the population level, the ratio of inhibition to excitation was higher during slow-wave-sleep up-states compared to the wake state. In 3 neurons that were recorded across several states, both average conductances together with their variances decreased in the wake state compared to slow-wave-sleep up-states. In addition, especially during the wake state, some cells displayed comparable excitation and inhibition or even a dominant excitation ( 2 out of 11 cells in the wake state). The study also reports an important diversity in the absolute values of the estimated conductance parameters.

An important concern in this type of studies is the estimation of the leak parameters of the recorded neurons. The down-states of the slow oscillation, when the local network is presumably silent, are too short for properly estimating these parameters, since these brief periods immediately following prolonged up-states are likely to include after-hyperpolarizing currents (Sanchez-Vives et al., 2000) that would bias the estimate of the leak. In both cases, values obtained from previous work (Paré et al, 1998; Destexhe and Paré, 1999) were used for the up-states: these studies evaluated that the ratio of input resistance after TTX block of synaptic activity to the total input resistance during up-states was about 4 to 6 -fold. The total input resistance was estimated in all cells studied from the linear portion of the I-V curve obtained during upstates. During wake or wake-like states, the mean total input resistance, as compared to the mean total input resistance during up-states, was taken into account to predict the ratio of input resistance with and without synaptic activity. The underlying hypothesis for these assumptions is that the ratio of leak conductance to synaptic conductance is similar in all cells during comparable network states, implying that the leak conductance and the total synaptic conductance covary in a strong way across cells. In the natural wakesleep cycle study, the dependence of the conductance estimates on this important ratio was systematically evaluated: this analysis showed that the estimated domination of inhibition was qualitatively robust, and that this prediction failed only when the leak conductance and the total synaptic conductance were assumed to be approximately equal.

On the other hand, the variance estimates do not depend on the leak conductance, provided that the total conductance is known, as is the case in the cited studies. They are, however, dependent on the membrane capacitance $C$ (see Eqs. 9 and 10). This value was assumed to be constant across the cells analyzed in vivo (supposing a specific membrane capacitance of $1 \mu \mathrm{F} / \mathrm{cm}^{2}$ and a membrane area of around $30000 \mu \mathrm{m}^{2}$ ). In future studies, it could possibly be estimated using short current pulse injection during the spontaneous activity, where the capacitance can be extracted from the time constant of exponential fits to the $\mathrm{V}_{m}$ decay. Synaptic conductance variances are useful parameters that can be related to spike initiation (see Sections 3.3 and 3.4). The $\mathrm{VmD}$ method provided the first published estimates for these parameters in vivo (for a different approach, see Monier et al., 2008, this issue). 


\subsection{Estimating time constants from $\mathbf{V}_{m}$ power spectral density}

\subsubsection{Outline of the method}

The point-conductance model given by Eqs. 13 was studied further, and recently we showed that the power spectral density (PSD) of the $\mathrm{V}_{m}$ fluctuations described by this model can be well approximated by the following expression (Destexhe and Rudolph, 2004):

$$
S_{V}(\omega)=\frac{4}{G_{T}^{2}} \frac{1}{1+\omega^{2} \tau_{m}^{2}}\left[\frac{\sigma_{e}^{2} \tau_{e}\left(E_{e}-\bar{V}\right)^{2}}{1+\omega^{2} \tau_{e}^{2}}+\frac{\sigma_{i}^{2} \tau_{i}\left(E_{i}-\bar{V}\right)^{2}}{1+\omega^{2} \tau_{i}^{2}}\right]
$$

where $\omega=2 \pi f, f$ is the frequency, $G_{T}=G_{L}+g_{e 0}+g_{i 0}$ is the total membrane conductance, $\tilde{\tau}_{m}=C / G_{T}$ is the effective time constant, and $\bar{V}=\left(G_{L} E_{L}+g_{e 0} E_{e}+g_{i 0} E_{i}\right) / G_{T}$ is the average membrane potential. The "effective leak" approximation used to derive this equation consisted in incorporating the average synaptic conductances into the total leak conductance, and then considering that fluctuations around the obtained mean voltage are subjected to a constant driving force (Destexhe and Rudolph, 2004).

As mentioned above, the synaptic time constant parameters, $\tau_{e}$ and $\tau_{i}$, need to be estimated in order to extract precise values of the conductance variances with the $\mathrm{VmD}$ method. As those two parameters appear in the theoretical expression of the $\mathrm{V}_{m}$ PSD, we explored the possibility of evaluating them from the analysis of the PSD of experimentally recorded $V_{m}$ fluctuations. To this end, the following simplified expression can be fitted:

$$
S_{V}(\omega)=\frac{1}{1+\omega^{2} \tilde{\tau}_{m}^{2}}\left[\frac{A_{e} \tau_{e}}{1+\omega^{2} \tau_{e}^{2}}+\frac{A_{i} \tau_{i}}{1+\omega^{2} \tau_{i}^{2}}\right],
$$

where $A_{e}$ and $A_{i}$ are amplitude parameters. This five parameter template is used to provide estimates of the parameters $\tau_{e}$ and $\tau_{i}$ (supposing that $\tilde{\tau}_{m}$ has been measured). A further simplification consists in assuming that $A_{e}=A_{i}$, which was used for fitting in vivo data (see Methods).

These analytic expressions were tested by comparing the prediction to numerical simulations of a singlecompartment model subject to fluctuating synaptic conductances (Eqs. 1 3). The matching between the analytic expression and the PSD obtained numerically was nearly perfect, as shown in Fig. $3 \mathrm{~A}$ and as reported in detail previously (Destexhe and Rudolph, 2004).

\subsubsection{Testing synaptic time constants estimates with dynamic-clamp}

We applied the procedure described above to the PSD of $\mathrm{V}_{m}$ fluctuations obtained by controlled, dynamicclamp fluctuating conductance injection in cortical neurons in vitro (using a new, high resolution electrode compensation technique, Brette et al., 2005, 2007; Rudolph et al., 2005). In this case, the scaling of the PSD conforms to the prediction (Fig. 3B): the theoretical template (Eq. 12) can provide a very good fit of the experimentally obtained PSD, up to around $400 \mathrm{~Hz}$, where recording noise becomes important. The template used was according to Eq. 11 or Eq. 12, both of which provided equally good fits (not shown). This shows that the analytic expression for the PSD is consistent not only with models, but also with conductance injection in real neurons in vitro. 


\subsubsection{PSD Analysis of $\mathbf{V}_{m}$ fluctuations in vitro and in vivo}

We have also attempted to apply the same procedure to $\mathrm{V}_{m}$ fluctuations resulting from real synaptic activity, during up-states recorded in vitro (Fig. 3C) and during sustained network activity in vivo (Fig. 3D). In this case, however, it is apparent that the experimental PSDs cannot be fitted with the theoretical template as nicely as for dynamic-clamp data (Fig. 3B). The PSD presents a frequency scaling region at high frequencies, and scales as $1 / f^{\alpha}$ with a different exponent $\alpha$ as predicted by the theory (see Figs. 3C-D). The analytic expression (Eq. 11 predicts that the PSD should scale as $1 / \mathrm{f}^{4}$ at high frequencies, but the experiments show that the exponent $\alpha$ is obviously lower than that value (see Discussion for possible reasons for such a difference). This difference of course compromises the accuracy of the method to estimate $\tau_{e}$ and $\tau_{i}$ in situations of real synaptic bombardment. Nevertheless, including the values of $\tau_{e}=3 \mathrm{~ms}$ and $\tau_{i}=10 \mathrm{~ms}$ provided acceptable fits to the low-frequency $(<100 \mathrm{~Hz})$ part of the spectrum (Fig. 3 C-D, red curves). However, in this case, small variations (around 20-30\%) around these values of $\tau_{e}$ and $\tau_{i}$ yielded equally good fits (not shown; see also Rudolph et al., 2005). Thus, the method cannot be used to precisely estimate those parameters, but can nevertheless be used to broadly estimate them with an error of the order of $30 \%$.

\subsection{Estimating spike-triggering conductance configurations}

\subsubsection{A preliminary investigation}

The conductance measurements outlined above show that there is a diversity of combinations of $g_{e}$ and $g_{i}$ that underlies the genesis of subthreshold activity in different preparations. We used a computational model based on the point-conductance model, but including a spiking mechanism (see Eq. 4), to reproduce those measurements and try to infer what different properties such states may have in terms of spike selectivity. Indeed, an infinite number of combinations of $g_{e}$ and $g_{i}$ can give similar $\mathrm{V}_{m}$ activity. Figure $4 \mathrm{~A}$ illustrates two extreme examples out of this continuum: first a state where both excitatory and inhibitory conductances are of comparable magnitude (Fig. 4A, left; "Equal conductances"). In this state, both conductances are lower than the resting conductance of the cell and the $\mathrm{V}_{m}$ is fluctuating around $-60 \mathrm{mV}$. Second, similar $\mathrm{V}_{m}$ fluctuations can be obtained when both conductances are of larger magnitude, but in this case, inhibition has to be augmented several-fold to maintain the $\mathrm{V}_{m}$ around $-60 \mathrm{mV}$ (Fig. 4A, right; "Inhibition-dominated"). Such conductance values are more typical of what is usually measured in vivo (Rudolph et al., 2005, 2007; Monier et al., 2008, this issue). Both conductances are larger than the resting conductance, a situation which can be described as a "high-conductance state".

To determine how these two states differ in their spike selectivity, we evaluated the spike-triggering conductances by averaging the conductance traces collected in $50 \mathrm{~ms}$ windows preceding spikes. This average pattern of conductance variations leading to spikes is shown in Fig. 4B. For equal-conductance states, there is an increase of total conductance preceding spikes (purple curve in Fig. 4B, left), as can be expected from the fact that excitation increases $\left(g_{e}\right.$ curve in Fig. $4 \mathrm{~B}$, left). In contrast, for inhibition-dominated states, the total conductance decreases prior to the spike (purple curve in Fig. $4 \mathrm{~B}$, right), and this decrease necessarily comes from a similar decrease of inhibitory conductance, which is, in this case, stronger than the increase of excitatory conductance ( $g_{i}$ curve in Fig. 4B, right). Thus, in such states the spike seems primarily caused by a drop of inhibition.

This pattern was seen not only in the average, but also at the level of single spikes. Using a vector representation to display the conductance variation preceding spikes (each vector links the conductance state in a 
window of 30-40 ms before the spike with that in the $10 \mathrm{~ms}$ preceding the spike) shows that the majority of spikes follow the average pattern (Fig. $4 \mathrm{C}$ ). The same features were also present when the integrate-and-fire model was used (not shown), and thus do not seem to depend on the spike generating mechanisms.

These patterns of conductance variations preceding spikes were also investigated in real neurons by using dynamic-clamp experiments to inject fluctuating conductances in vitro. In this case, performing the same analysis as above revealed similar features: spike-triggered averages (STAs) of the injected conductances displayed either increase or decrease in total conductance, depending on the conductance parameters used (Fig. 5A), and the vector representations were also similar (Fig. 5B). It suggested that these features are independent of the spike generating mechanism but rather are caused by subthreshold $\mathrm{V}_{m}$ dynamics.

\subsubsection{A geometrical interpretation based on the point-conductance model}

The configuration of synaptic conductances just before spikes can be explained qualitatively by considering that the total current must be positive at spike time, i.e., $g_{e}\left(E_{e}-V_{t}\right)+g_{i}\left(E_{i}-V_{t}\right)+G_{L}\left(E_{L}-V_{t}\right)>0$, where $V_{t}$ is the spike threshold (using an integrate-and-fire approximation).

This inequality defines a half-plane in which $\left(g_{e}, g_{i}\right)$ must lie at spike time. Fig. 6A shows graphically how this inequality affects the synaptic conductances. The variable $\left(g_{e}, g_{i}\right)$ is normally distributed, so that isoprobability curves are ellipses in the plane (plotted in red). In that plane, the line $\left\{g_{e}+g_{i}=g_{e 0}+\right.$ $\left.g_{i 0}\right\}$ going through the center of the ellipses defines the points for which the total conductance equals the mean conductance, and the line $\left\{g_{e}\left(E_{e}-V_{t}\right)+g_{i}\left(E_{i}-V_{t}\right)+G_{L}\left(E_{L}-V_{t}\right)=0\right\}$ defines the border of the half-plane in which conductances lie at spike time. In the equal conductances regime (Fig. 6A, left), synaptic conductances are small and have similar variances, so that isoprobability curves are circular; the intersection of the half-plane with those circles is mostly above the mean total conductance line, so that the total conductance is higher than average at spike time.

In the inhibition-dominated regime (Fig. 6A, right), synaptic conductances are large and the variance of $g_{i}$ is larger than the variance of $g_{e}$, so that isoprobability curves are vertically elongated ellipses; the intersection of the half-plane with those ellipses is essentially below the mean total conductance line, so that the total conductance is lower than average at spike time.

More precisely, when isoprobability curves are circular (equal variances), then the expected total conductance is unchanged at spike time when the lines $\left\{g_{e}\left(E_{e}-V_{t}\right)+g_{i}\left(E_{i}-V_{t}\right)+G_{L}\left(E_{L}-V_{t}\right)=0\right\}$ and $\left\{g_{e}+g_{i}=g_{e 0}+g_{i 0}\right\}$ are orthogonal, i.e., when $E_{e}-V_{t}+E_{i}-V_{t}=0$. Spikes are associated with increases in conductance when the first line has a higher slope, i.e., when $E_{e}-V_{t}>V_{t}-E_{i}$ (which is typically the case).

When isoprobability curves are not circular, we can look at the graph in the space $\left(\frac{g_{e}}{\sigma_{e}}, \frac{g_{i}}{\sigma_{i}}\right)$ where isoprobability curves are circular. Then the orthogonality condition between the lines

$$
\left\{\frac{g_{e}}{\sigma_{e}} \sigma_{e}\left(E_{e}-V_{t}\right)+\frac{g_{i}}{\sigma_{i}} \sigma_{i}\left(E_{i}-V_{t}\right)+G_{L}\left(E_{L}-V_{t}\right)=0\right\}
$$

and

$$
\left\{\frac{g_{e}}{\sigma_{e}} \sigma_{e}+\frac{g_{i}}{\sigma_{i}} \sigma_{i}=g_{e 0}+g_{i 0}\right\}
$$

reads

$$
\sigma_{e}^{2}\left(E_{e}-V_{t}\right)+\sigma_{i}^{2}\left(E_{i}-V_{t}\right)=0
$$


It follows that spikes are associated with increases in total conductance when the following condition is met:

$$
\frac{\sigma_{e}}{\sigma_{i}}>\sqrt{\frac{V_{t}-E_{i}}{E_{e}-V_{t}}} .
$$

One can also recover this result by calculating the expectation of the conductance change conditionally to the fact that the current at spike threshold is positive (implicitly, we are neglecting the correlation time constants of the synaptic conductances). Using typical values ( $\left.V_{t}=-55 \mathrm{mV}, E_{e}=0 \mathrm{mV}, E_{i}=-75 \mathrm{mV}\right)$, we conclude that spikes are associated with increases in total conductance when $\sigma_{e}>0.6 \sigma_{i}$. This inequality is indeed satisfied in the equal conductances regime and not in the inhibition-dominated regime investigated above.

\subsubsection{Testing the geometrical prediction with dynamic-clamp}

The geometrical reasoning predicts that the sign of the total synaptic conductance change triggering spikes depends only on the ratio of synaptic variances, and not on the average conductances. We have systematically tested this prediction using dynamic-clamp injection of fluctuating conductances in vitro. In 8 regular spiking cortical neurons, we scanned different parameter regimes in a total of 36 fluctuating conductance injections. Fig. 6B shows two examples from the same cell: both correspond to an average "high conductance" regime, dominated by inhibition, but in one case it is the variance of excitation, in the other case the variance of inhibition, that is higher. We can see that the total conductance before the spike increases in the first case, but decreases in the second. Fig. 6C (left) shows the average total conductance change preceding spikes as a function of $\sigma_{e} / \sigma_{i}$, for all the 36 injections: the vertical dashed line represents the predicted value of $\sigma_{e} / \sigma_{i}=0.6$, which indeed separates all the "conductance drop" configurations from the "conductance increase" configurations. Even though the prediction is based on a simple integrate-and-fire extension of the point-conductance model, we can see that the ratio of synaptic variances can predict the sign of the total conductance change triggering spikes in biological cortical neurons subjected to fluctuating excitatory and inhibitory conductances.

In addition (Fig. 6C, right), the dynamic-clamp data shows that the average amplitude of change $\left(\Delta g_{e}=\right.$ $g_{e 0} k_{e}$, see Methods) of each synaptic conductance preceding a spike is related, in a linear way, to the standard deviation of this conductance. For a fixed value of standard deviation, there was no significant influence of the average conductance (not shown). This observation is consistent with the idea that in all the cases studied here, the firing of the cell was driven by fluctuations in the $\mathrm{V}_{m}$, rather than by a high mean $\mathrm{V}_{m}$ value (not shown).

Taken together, these theoretical and experimental analyses indicate that the average total conductance drop preceding spikes, as seen in the "high conductance" case we initially considered (Fig. 4), is not a direct consequence of the "high conductance" state of the membrane, but is in fact related to the high inhibitory variance, which is indeed to be expected especially when the mean inhibitory conductance is also high (as confirmed by the studies presented in the first part of this article).

\subsection{Estimating spike-triggered averages of synaptic conductances from the $V_{m}$}

In order to extend the analysis of spike-triggering conductance configurations to real recurrent cortical activity observed in vivo, we recently developed a procedure to extract the spike-triggered averages (STAs) 
of conductances from recordings of the $\mathrm{V}_{m}$ (Fig. 7) Pospischil et al., 2007). The STA of the $\mathrm{V}_{m}$ is calculated first, and the method searches for the "most likely" spike-related conductance time courses $\left(g_{e}(t), g_{i}(t)\right)$ that are compatible with the observed voltage STA. The procedure is based on a discretization of the time axis in Eqs. 1-3, which, rearranged, lead to the following relations:

$$
\begin{aligned}
g_{i}^{k} & =-\frac{C}{V^{k}-E_{i}}\left\{\frac{V^{k}-E_{L}}{\tau_{L}}+\frac{g_{e}^{k}\left(V^{k}-E_{e}\right)}{C}+\frac{V^{k+1}-V^{k}}{\Delta t}-\frac{I_{e x t}}{C}\right\}, \\
\xi_{e}^{k} & =\frac{1}{\sigma_{e}} \sqrt{\frac{\tau_{e}}{2 \Delta t}}\left(g_{e}^{k+1}-g_{e}^{k}\left(1-\frac{\Delta t}{\tau_{e}}\right)-\frac{\Delta t}{\tau_{e}} g_{e 0}\right) \\
\xi_{i}^{k} & =\frac{1}{\sigma_{i}} \sqrt{\frac{\tau_{i}}{2 \Delta t}}\left(g_{i}^{k+1}-g_{i}^{k}\left(1-\frac{\Delta t}{\tau_{i}}\right)-\frac{\Delta t}{\tau_{i}} g_{i 0}\right)
\end{aligned}
$$

where $\tau_{L}=C / G_{L}$ is the resting membrane time constant. Note that $\xi_{e}(t)$ and $\xi_{i}(t)$ have become Gaussiandistributed random numbers $\xi_{e}^{k}$ and $\xi_{i}^{k}$. There is a continuum of combinations $\left\{g_{e}^{k+1}, g_{i}^{k+1}\right\}$ that can advance the membrane potential from $V^{k+1}$ to $V^{k+2}$, each pair occurring with a probability

$$
\begin{aligned}
p^{k}:= & p\left(g_{e}^{k+1}, g_{i}^{k+1} \mid g_{e}^{k}, g_{i}^{k}\right)=\frac{1}{2 \pi} e^{-\frac{1}{2}\left(\xi_{e}^{k 2}+\xi_{i}^{k 2}\right)}=\frac{1}{2 \pi} e^{-\frac{1}{4 \Delta t} X^{k}}, \\
X^{k}= & \frac{\tau_{e}}{\sigma_{e}^{2}}\left(g_{e}^{k+1}-g_{e}^{k}\left(1-\frac{\Delta t}{\tau_{e}}\right)-\frac{\Delta t}{\tau_{e}} g_{e 0}\right)^{2} \\
& +\frac{\tau_{i}}{\sigma_{i}^{2}}\left(g_{i}^{k+1}-g_{i}^{k}\left(1-\frac{\Delta t}{\tau_{i}}\right)-\frac{\Delta t}{\tau_{i}} g_{i 0}\right)^{2} .
\end{aligned}
$$

Because of Eq. 13, $g_{e}^{k}$ and $g_{i}^{k}$ are not independent and $p^{k}$ is, thus, a unidimensional distribution only. Given initial conductances $\left\{g_{e}^{0}, g_{i}^{0}\right\}$, one can write down the probability $p$ for certain series of conductances $\left\{g_{e}^{j}, g_{i}^{j}\right\}_{j=0, \ldots, n}$ to occur that reproduce a given voltage trace $\left\{V^{l}\right\}_{l=1, \ldots, n+1}$ :

$$
p=\prod_{k=0}^{n-1} p^{k}
$$

Due to the symmetry of the distribution $p$, the average paths of the conductances coincide with the most likely ones. It is thus sufficient to determine the conductance series with extremal likelihood by solving the n-dimensional system of linear equations

$$
\left\{\frac{\partial X}{\partial g_{e}^{k}}=0\right\}_{k=1, \ldots, n},
$$

where $X=\sum_{k=0}^{n-1} X^{k}$, for the vector $\left\{g_{e}^{k}\right\}$. This is equivalent to solving $\left\{\frac{\partial p}{\partial g_{e}^{k}}=0\right\}_{k=1, \ldots, n}$ and involves the numerical inversion of an $n \times n$-matrix, which can be done using standard numeric methods (Press et al., 1986). The series $\left\{g_{i}^{k}\right\}$ is subsequently obtained from Eq. 13 . Details of this procedure as well as an evaluation of its performance can be found in Pospischil et al., 2007.

The method requires first an estimation of the parameters describing the distribution of each of the conductances, which can be obtained by the $\mathrm{VmD}$ method. The leak parameters of the cell, or alternatively the effective parameters during $\mathrm{V}_{m}$ fluctuations (effective conductance and effective time constant), also 
have to be estimated prior to this analysis. The method was successfully tested using computational models: conductance STAs extracted from the $\mathrm{V}_{m}$ of an integrate-and-fire point-conductance model are nearly identical to the numerically obtained conductance STAs (Fig. 7A, left; Pospischil et al., 2007).

During response to sensory stimuli, there can be a substantial degree of correlation between excitatory and inhibitory synaptic input (Monier et al., 2003; Wehr and Zador, 2003; Wilent and Contreras, 2005). Since this situation has not been addressed in Pospischil et al., 2007, we would like to sketch a possible extension of the method. To this end, we reformulate the discretized versions of Eqs. 2, 3 in the following way:

$$
\begin{aligned}
\frac{g_{e}^{k+1}-g_{e}^{k}}{\Delta t} & =-\frac{g_{e}^{k}-g_{e 0}}{\tau_{e}}+\sigma_{e} \sqrt{\frac{2 \Delta t}{\tau_{e}(1+c)}}\left(\xi_{1}^{k}+\sqrt{c} \xi_{2}^{k}\right), \\
\frac{g_{i}^{k+1}-g_{i}^{k}}{\Delta t} & =-\frac{g_{i}^{k}-g_{i 0}}{\tau_{i}}+\sigma_{i} \sqrt{\frac{2 \Delta t}{\tau_{i}(1+c)}}\left(\xi_{2}^{k-d}+\sqrt{c} \xi_{1}^{k-d}\right) .
\end{aligned}
$$

Here, instead of having one "private" white noise source feeding each conductance channel, now the same two noise sources $\xi_{1}$ and $\xi_{2}$ contribute to both inhibition and excitation. The amount of correlation is tuned by the parameter $c$. Also, since there is evidence that the peak of the $g_{e}-g_{i}$-crosscorrelation is not always centered at 0 during stimulus-evoked responses ("delayed inhibition"; see Wehr and Zador, 2003; Wilent and Contreras, 2005), we allow a non-zero delay $d$ : for a positive parameter $d$, the inhibitory channel receives the input that the excitatory channel received $d$ time steps before. Eqs. 20 and 21 can be solved for $\xi_{1}^{k}$ and $\xi_{2}^{k}$, thus replacing Eqs. 14 and 15 . It is then possible to proceed as in the uncorrelated case, where now, due to the delay, the matrix describing Eq. 19 has additional subdiagonal entries.

However, the application of this extended method requires the estimation of the usual leak parameters, of conductance distribution parameters - for which the $\mathrm{VmD}$ method cannot be directly used in its current form since it is based on uncorrelated noise sources - as well as knowledge of the parameters $c$ and $d$. At present, we can only speculate on how $c$ and $d$ could be evaluated in experiments: extracellularly recorded spike trains could perhaps be used to this end, provided that simultaneously recorded single units could be classified as excitatory or inhibitory. Alternatively, different plausible $c$ and $d$ values could be scanned to examine how they could potentially influence the conductance STAs extracted from a given $\mathrm{V}_{m}$ STA.

\subsubsection{Testing STA estimation with dynamic-clamp}

The dynamic-clamp data presented above was used to evaluate the accuracy of the conductance STA estimation method (for the uncorrelated case only): indeed, the conductance STAs estimated from the $\mathrm{V}_{m}$ STAs could be compared to conductance STAs obtained directly by averaging conductance traces, since in dynamic-clamp the injected conductances are perfectly controlled by the experimentalist. For the 36 injections analyzed, a good match was observed between the two (see one example in Fig. 74, right panel). Since we intended to evaluate the STA method specifically, we assumed that conductance distribution parameters were known. In addition, we estimated the cell's leak parameters (see Methods). To quantify the comparison on a population basis, we did the following analyses: exponential functions (see Methods) were fitted to each conductance STA (estimated and directly measured) starting at $1 \mathrm{~ms}$ before the spike and decaying to baseline backwards in time. We then compared the asymptotic values (i.e., the average baseline conductances) and the time constants of these fits, as well as the amplitude of conductance change from the start of the fit to the asymptote (i.e., the amplitude of conductance change preceding the spike). In all cases but one, the exponential functions provided excellent fits to the conductance STAs. It was neces- 
sary to exclude the $1 \mathrm{~ms}$ time window preceding the spike to avoid severe contamination of the analyses by intrinsic conductances. Excluding a broader time window did not improve the analyses for these neurons.

The average baseline conductances always matched very well (error of $-0.1 \pm 0.25 \mathrm{nS}$, or $-0.8 \pm 2.6 \%$, for excitation; $0.8 \pm 1.5 \mathrm{nS}$, or $0.6 \pm 4.5 \%$, for inhibition; not shown). More importantly, the estimates of the average conductance patterns leading to spikes were also in good correspondence with the measured patterns, in terms of both the amplitude of conductance change (Fig. 7 $\mathrm{C}$, top; error of $-1.2 \pm 3 \mathrm{nS}$, or -26 $\pm 28.8 \%$, for excitatory amplitude change, and $-2.0 \pm 2.5 \mathrm{nS}$, or $-10.7 \pm 47 \%$, for inhibitory amplitude change) and the time constant (Fig. 7C, bottom; error of $0.39 \pm 0.48 \mathrm{~ms}$, or $11.2 \pm 21.1 \%$ for excitatory time constant, and $0.36 \pm 1.71$, or $2.6 \pm 18.8 \%$, for inhibitory time constant). For excitation, the error on the estimate of the amplitude is correlated with the error on the estimate of the time constant (not shown): this suggests that, in most cases, a slightly too fast rise of the excitatory conductance results in a slightly too high amplitude of conductance change. The lack of correlation between the two error measures in the case of the inhibitory conductance points to a more complex origin for the observed errors. Moreover, the errors on the amplitude of the two conductance changes are positively correlated (Fig. 7D, top). This dependency actually ensures that the error on the estimated total conductance change (excitation-inhibition) remains small (-0.8 $\pm 2.4 \mathrm{nS}$; Fig. 7D, bottom).

Finally, we have investigated the dependency of the estimate errors on a diversity of variables, and found a correlation of amplitude errors with the average $\mathrm{V}_{m}$ during the fluctuating conductance injection (not shown): this dependency points to a possible contamination by intrinsic conductances activated differentially at different average $\mathrm{V}_{m}$ levels. However, we found no dependency of the error on average firing rate (for the rates up to around $30 \mathrm{~Hz}$ studied here), suggesting no important contamination by spike-dependent conductances like the ones underlying the after-hyperpolarization (when care is taken to compute STAs using spikes preceded by at least $100 \mathrm{~ms}$ of silence, see Methods).

We have also verified that conductance STA estimates can be relied on to investigate what factors determine the average conductance variations preceding spikes. Fig. 8 shows that the analyses performed previously on dynamic-clamp data (i.e., on STAs obtained directly by averaging the conductance traces, Fig. 6B-C), can also be successfully performed using the conductance STAs estimated from the corresponding $\mathrm{V}_{m}$ STAs: Fig. 8A shows the estimated STAs in the two different "high conductance" states, dominated by either excitatory or inhibitory variance (compare to Fig. 6B). Fig. $8 \mathrm{~B}$ shows, for the population data, the (significant) correlations between total conductance change and $\sigma_{e} / \sigma_{i}$, as well as between the change of each of the conductances and the corresponding standard deviation. Note the similarity between Fig. 6B-C and Fig. 8A-B, even though the correlations at the population level are more noisy when the estimated STAs are used.

\subsubsection{STA Analysis of intracellular recordings of cortical neurons in vivo}

The conductance STA estimation method was used to determine conductance variations preceding spikes during $\mathrm{V}_{m}$ fluctuations in vivo (Rudolph et al., 2007). Starting from $\mathrm{V}_{m}$ recordings of spontaneous spiking activity in awake or naturally sleeping cats, we computed the spike-triggered average of the $\mathrm{V}_{m}$ (Fig. 9). Using values of $g_{e 0}, g_{i 0}, \sigma_{e}, \sigma_{i}$ estimated using the $\mathrm{VmD}$ method (see above), we computed the most likely conductance traces yielding the observed $\mathrm{V}_{m}$ averages. Most of these analyses ( 7 out of 10 cells for awake, 6 out of 6 for slow-wave-sleep, 2 out of 2 for REM) revealed conductance dynamics consistent with states dominated by inhibitory variance: there was a drop of the total conductance preceding spikes, due to a strong decrease of the inhibitory conductance (Fig. 9, right). However, a few cases, in the wake state (3 out of 10 cells), displayed the opposite configuration with the total synaptic conductance increasing before the 
spike (Fig. 9, left).

We also checked how the geometrical prediction relating the sign of total conductance change preceding spikes and the ratio $\sigma_{e} / \sigma_{i}$ performed for this data (Fig. 9B). We have seen that the critical value of $\sigma_{e} / \sigma_{i}$ for which the total conductance change shifts from positive to negative depends on the spike threshold. This parameter was quite variable in the recorded cells, and so a critical $\sigma_{e} / \sigma_{i}$ value was calculated for each cell. Fig. 9B shows the lowest and highest critical values obtained (dashed lines), and also displays in white the cells which do not conform to the prediction based on their critical value. This is the case for only 4 out of 18 cells, for three of which the total conductance change is close to zero.

The extraction of conductance STAs depends on the accuracy of the synaptic conductance parameters estimated with the $\mathrm{VmD}$ method, which means that assumptions made about the leak conductance and the cell capacitance will influence the results (see Section 3.1.3). We have shown that the ratio $\sigma_{e} / \sigma_{i}$ should determine whether, on average, the total conductance increases or decreases prior to the spike. This ratio is independent of the leak conductance, but it depends on the capacitance $C$. However, it appears in both the numerator and the denominator of the ratio. Fig. $9 \mathrm{C}$ shows the dependency of $\sigma_{e} / \sigma_{i}$ on this parameter for different values of total input resistance and two sets of realistic values for the $\mathrm{V}_{m}$ distribution parameters. This analysis indicates that a reasonable error on $C$ produces a limited error on the ratio $\sigma_{e} / \sigma_{i}$, and suggests that conclusions drawn from the in vivo data about the respective contributions of excitation and inhibition in triggering spikes are valid.

\section{Discussion}

\subsection{Synaptic conductance analysis methods based on the fluctuating point-con- ductance model}

We presented how the simple point-conductance model of cortical synaptic activity can provide a basis for the analysis of experimental data, essentially through the matching of expressions derived from the model to intracellular $\mathrm{V}_{m}$ recordings of cortical neurons. This approach has been used for extracting different parameters from the recurrent cortical activity in vivo: the averages and variances of excitatory and inhibitory conductances, their decay time constants and the optimal conductance waveform underlying spike selectivity. These analyses were possible because the point conductance model represents in a compact and mathematically tractable way the activity resulting from several thousand synapses.

The $\mathrm{VmD}$ analysis provides a characterization of synaptic activity in simple terms (average conductance, level of fluctuations). Such parameters can readily be incorporated in computational models to yield the $\mathrm{V}_{m}$ and conductance state corresponding to in vivo activity with just a few variables. This approach has been used for example in network simulations to obtain realistic conductance states in neurons even with small networks (Haeusler and Maass, 2007). It is also directly usable in dynamic-clamp experiments to investigate the impact of synaptic background activity on signal processing by single cortical or thalamic neurons (Fellous et al., 2003; Shu et al., 2003; Wolfart et al., 2005; Desai and Walcott, 2006).

Beyond the matching of experimental $\mathrm{V}_{m}$ distributions to a theoretical expression ( $\mathrm{VmD}$ method), we have also attempted to match the PSDs of $\mathrm{V}_{m}$ fluctuations. This approach provides some validation for assumptions made about synaptic time constants on the basis of published studies (Destexhe and Paré, 1999; Destexhe et al., 2001). However, the fact that the point-conductance model does not account for 
the scaling properties of experimental PSDs (Fig. 3C-D) limits the accuracy of the method and yields only broad estimates of the synaptic time constants (approximately 30\% error). A parallel study (Bedard and Destexhe, 2007) has shown that the frequency scaling observed experimentally cannot be accounted for by standard cable theory, and modifications of cable equations are required to match those values. We hope to obtain a more accurate fitting template, which would allow more precise PSD analyses in the future.

Finally, in order to be able to study average spike-triggered patterns of conductances in vivo, we have recently developed a probabilistic method for extracting the STAs of conductances from STAs of the $\mathrm{V}_{m}$ (Pospischil et al., 2007). This method relies on the estimation of a number of parameters inherent to the neuron (like leak conductance and capacitance), as well as on synaptic conductance parameters estimated, for example, with the $\mathrm{VmD}$ method. We provided new data from dynamic-clamp experiments in vitro, demonstrating that given the synaptic conductance parameters, a good match between the extracted conductance STAs and the actual, known conductance STAs can be obtained.

\subsection{Comparison to other synaptic conductance analysis methods}

Other studies proposed synaptic conductance estimates in vivo derived from I-V curves (in current-clamp) or V-I curves (in voltage-clamp) obtained at different points in time following a stimulus (Borg-Graham et al.,1998; Anderson et al., 2000; Monier et al., 2003; Wehr and Zador, 2003; Wilent and Contreras, 2005) or the onset of an up-state (Haider et al., 2006). An exhaustive comparison of these studies is beyond the scope of the present article (see Monier et al., 2008, this issue), but a few points can be stressed. The fact that the VmD method applies to current-clamp data obtained at a few levels of constant injected current circumvents technical problems with voltage-clamp due to high series resistance of patch electrodes in vivo (but see Borg-Graham et al., 1998; Monier et al., 2003; Wehr and Zador, 2005) or the need for discontinuous voltage-clamp with sharp electrodes (Haider et al., 2006). The fact that it relies on a strong assumption about the stochasticity of synaptic inputs and the independence of excitation and inhibition allows the analysis of spontaneous cortical activity with no "zero" time point. However, this assumption also makes it unsuited, in its present form, for the analysis of stimulus-evoked activity with important temporal structure.

The VmD method suffers from one common limitation with other approaches for synaptic conductance estimation: the need to separate synaptic currents from leak currents. The fact that it is used for the analysis of on-going, spontaneous activity in cortical networks poses, however, an additional complication: indeed, when synaptic inputs evoked by sensory stimulation are analyzed, it is with reference to the pre-stimulus activity, which includes the leak current and any other baseline currents, including on-going synaptic activity (Borg-Graham et al.,1998; Anderson et al., 2000; Monier et al., 2003; Wehr and Zador, 2003; Wilent and Contreras, 2005). When we attempt to analyze the spontaneous activity itself, there is no straightforward reference that can be used. As mentioned above, the short down-states do not seem a good candidate since they include after-hyperpolarizing currents consecutive to the up-states (Sanchez-Vives et al., 2000), although a down-state-referenced analysis (as in Haider et al., 2006) could perhaps be compared to the analysis performed in the reviewed studies. However, in the wake state, the continuous on-going activity does not even present down-states. The only precise approach to evaluate the leak conductance for each studied cell is to block all synaptic activity with TTX, but that also means recording only one or two cells per animal for this protocol, which is an extremely constraining experimental situation. The compromise chosen in the reviewed in vivo studies consisted in using published average values obtained in previous TTX experiments in a similar preparation (Paré et al., 1998) and checking the robustness of the estimates to the assumed leak conductance parameter. The estimates should be re-evaluated in the light of any future 
experimental data providing information about the leak parameters of cortical cells in vivo in the absence of synaptic activity. Optimally, such future studies would include the simple, classical protocol required for a subsequent $\mathrm{VmD}$ analysis: several-second-long current-clamp recordings of the $\mathrm{V}_{m}$ at different levels of steady injected current.

As to our probabilistic method for extracting the STAs of conductances from STAs of the $\mathrm{V}_{m}$ (Pospischil et al., 2007), we are not aware of any other method currently allowing this analysis for spontaneous activity: in voltage-clamp, no spikes are recorded and so, obviously, conductances leading to spikes cannot be extracted directly. Voltage-clamp can only be used for extracting plausible conductance STAs when the precise times of spikes are known and reproducible (for example, at a given delay after a sensory stimulation), so that the estimated, stimulus-locked conductance dynamics can be reasonably expected to lead to spikes in the current-clamp configuration (Monier et al., 2003; Wehr and Zador, 2003).

\subsection{Dependency of the STA extraction method on the VmD method}

The result of the STA analysis is, however, dependent on the estimates of synaptic averages and variances, so that we may ask to what extent this result depends on the accuracy of the VmD method. In general, the average baseline conductances will reflect the estimates of average synaptic conductances (since both analyses are constrained by the same total input resistance measure and the same leak conductance assumption), unless the total input resistance changes markedly between the current levels used for the VmD analysis and the zero-current level at which STAs are extracted, due to activation of intrinsic conductances: in this case, since the conductances $\left(G_{L}, g_{e}(t), g_{i}(t)\right)$ have to be compatible with the voltage $V_{m}(t)$, the conductance STA baselines can be considerably shifted away from the mean conductance values tens of ms before the spike. The same effect is seen in in vitro dynamic-clamp experiments when a wrong leak conductance value is used (not shown). This distortion could be used as an indication for important activation of intrinsic conductances and suggest that the result should be discarded.

The variances of the synaptic conductances are correlated to the amplitudes of average conductance change preceding a spike (as shown in Fig. 6). The estimation of synaptic conductance variances with the VmD method is independent of the assumption made about the leak conductance, which excludes this source of potential error. It is dependent on the membrane capacitance $C$, which should be evaluated on a cellby-cell basis whenever possible in future studies. However, we have also shown that the ratio of synaptic conductance variances is only weakly dependent on the precise value of $C$ : this ratio determines the sign of total conductance change preceding a spike, so that the estimates of this sign from in vivo data seem robust (see below).

\subsection{Dynamic-clamp as a tool to evaluate conductance analysis methods}

We illustrated how and to what extent the validity of different approaches for conductance analysis can be tested using dynamic-clamp. This electrophysiological technique is an attractive tool to evaluate methods of conductance analysis, since it allows to mimic the activation of known conductances in a biological neuron: the results of an analysis method based on $\mathrm{V}_{m}$ recordings can be directly compared to measures of the actual conductances controlled by the experimentalist. In all the dynamic-clamp applications presented here, we have used the same description for the synaptic conductances - the Ornstein-Uhlenbeck stochastic model as in the theoretical analyses. This means that we could compare how the analysis methods perform if the stochastic conductances are inserted at the soma of a real cortical neuron, with a complex structure and a 
variety of intrinsic channels, instead of a passive single compartment. As we have seen, potential dendritic effects solicited only during distributed synaptic stimulation, like, possibly, the unexpected scaling of the $\mathrm{V}_{m}$ PSDs during real synaptic activity (Bedard and Destexhe, 2007), cannot be addressed with this somatic injection technique. They could perhaps be investigated in the future using dendritic patch-clamp.

The comparison was performed most extensively for the conductance STA estimation method: it indicates that if the window of analysis is chosen properly (by excluding a window of about $1 \mathrm{~ms}$ before the spike, and also excluding inter-spike-intervals shorter than around $100 \mathrm{~ms}$ ), the estimations perform well, and that the estimation errors, correlated with the average $\mathrm{V}_{m}$, are presumably linked to $\mathrm{V}_{m}$-dependent intrinsic channels. However, we did not systematically compare how the methods perform if the synaptic conductances deviate from the Ornstein-Uhlenbeck model: such an approach could constitute another application of the dynamic-clamp tool to the evaluation of conductance analysis methods. We have also not attempted yet to evaluate in dynamic-clamp the extended STA analysis method sketched above, which incorporates a known correlation between excitation and inhibition (Eqs. 20,21).

\subsection{Patterns of excitation and inhibition triggering spikes depend on the variances of the synaptic inputs}

What are the patterns of excitation and inhibition triggering spikes under different conditions of network activity ? A preliminary study, using both models and dynamic-clamp conductance injection in vitro, pointed to the fact that these patterns depend on the statistics of synaptic conductances, and that spikes could be preceded, on average, either by increases in total synaptic conductance, indicating a predominant role for excitation, or by decreases in total synaptic conductance, indicating a predominant role for inhibition. Other authors (Hasenstaub et al., 2005) have recently suggested that drop of inhibition can play an important role in determining spike timing in cortical neurons, based on dynamic-clamp injection of specific synaptic conductances with parameters matched to their in vivo recordings. Here we explored this issue further and showed, first by a theoretical reasoning, and second by scanning different parameter regimes using dynamic-clamp conductance injection, that the sign of the total conductance change before a spike does not directly depend on the average synaptic conductances, but is solely determined by the ratio of synaptic conductance variances. The variance of synaptic conductances in the Ornstein-Uhlenbeck model is related to the degree of correlation between Poisson input trains of each type in a detailed biophysical model (Destexhe et al., 2001): the level of synchrony among inhibitory neurons could thus be determining for spike timing whenever it significantly exceeds the level of synchrony among excitatory neurons. The rule relating the ratio of synaptic conductance variances with the sign of the average conductance change preceding a spike appears as a generalization of more specific results obtained by Tateno and Robinson in cortical neurons (Tateno and Robinson, 2006), as well as by the group of Jaeger in the cerebellum (Gauck and Jaeger, 2000, 2003; Suter and Jaeger, 2004): these authors also used dynamic-clamp injection of synaptic conductances and the conductance STAs they obtained seem consistent with our rule.

This result stresses the importance of evaluating the variances of synaptic conductances, in addition to their averages, when analyzing $\mathrm{V}_{m}$ fluctuations recorded in vivo. To our knowledge, the two reviewed studies using the VmD method (Rudolph et al., 2005, 2007) are the only ones (together with Monier et al., 2008, this issue) explicitely providing estimates of synaptic conductance variances. The application of the probabilistic method also allowed for the first time the extraction of synaptic conductance STAs from in vivo recordings of spontaneous $\mathrm{V}_{m}$ fluctuations in awake and naturally sleeping cats (Rudolph et al., 2007), and led to the observation of both types of firing regimes described above - average total conductance increase and average total conductance drop - with a majority of cases displaying the inhibition-dominated, 
conductance-drop pattern.

\section{Acknowledgments}

We thank Igor Timofeev for giving us permission to use some of his data. Research supported by CNRS, ANR, ACI, HFSP and the European Community (FACETS grant FP6 15879). Z.P. gratefully acknowledges the support of the FRM.

\section{References}

Anderson JS, Carandini M, Ferster D. Orientation tuning of input conductance, excitation, and inhibition in cat primary visual cortex. J Neurophysiol 2000;84:909-26.

Barak O, Tsodyks M. Persistent activity in neural networks with dynamic synapses. PLoS Comput Biol 2007;3:e35.

Baranyi A, Szente MB, Woody CD. Electrophysiological characterization of different types of neurons recorded in vivo in the motor cortex of the cat. II. Membrane parameters, action potentials, current-induced voltage responses and electrotonic structures. J Neurophysiol 1993;69:1865-79.

Bedard C, Destexhe A. A modified cable formalism for modeling neuronal membranes at high frequencies. Biophys. J. 2007; in press. Preprint available at http://arxiv.org/abs/0705.3759.

Bernander O, Douglas RJ, Martin KA, Koch C. Synaptic background activity influences spatiotemporal integration in single pyramidal cells. Proc Natl Acad Sci USA 1991;88:11569-73.

Borg-Graham LJ, Monier C, Frégnac Y. Visual input evokes transient and strong shunting inhibition in visual cortical neurons. Nature 1998;393:369-73.

Brette R, Rudolph M, Piwkowska Z, Bal T, Destexhe A. How to emulate double-electrode recordings with a single electrode? A new method of active electrode compensation. Soc Neurosci Abstracts 2005;688.2

Brette R, Piwkowska Z, Rudolph M, Bal T, Destexhe, A. A nonparametric electrode model for intracellular recording. Neurocomputing 2007;70:1597-601.

Burkitt AN, Meffin H, Grayden DB. Study of neuronal gain in a conductance-based leaky integrate-and-fire neuron model with balanced excitatory and inhibitory synaptic input. Biol. Cybern. 2003;89:119-25.

Compte A, Sanchez-Vives MV, McCormick DA, Wang XJ. Cellular and network mechanisms of slow oscillatory activity $(<1 \mathrm{~Hz})$ and wave propagations in a cortical network model. J Neurophysiol 2003;89:2707-25.

Cunningham MO, Pervouchine DD, Racca C, Kopell NJ, Davies CH, Jones RS, Traub RD, Whittington MA. Neuronal metabolism governs cortical network response state. Proc Natl Acad Sci USA 2006;103:5597-601.

Desai NS, Walcott EC. Synaptic bombardment modulates muscarinic effects in forelimb motor cortex. J Neurosci 2006;26:2215-26.

Destexhe A and Paré D. Impact of network activity on the integrative properties of neocortical pyramidal neurons in vivo. J Neurophysiol 1999;81:1531-47. 
Destexhe A, Badoual M, Piwkowska Z, Bal T, Hasenstaub A, Shu Y, McCormick DA, Pelletier J, Paré D, Rudolph M. In vivo, in vitro and computational evidence for balanced or inhibition-dominated network states, and their respective impact on the firing mode of neocortical neurons. Soc. Neurosci. Abstracts 2003a;29:921.14

Destexhe A, Rudolph M. Extracting information from the power spectrum of synaptic noise. J Comput Neurosci 2004;17:327-45.

Destexhe A, Hughes SW, Rudolph M, Crunelli V. Are corticothalamic "up" states fragments of wakefulness? Trends Neurosci 2007; doi:10.1016/j.tins.2007.04.006

Destexhe A, Rudolph M, Fellous J-M, Sejnowski TJ. Fluctuating synaptic conductances recreate in vivo-like activity in neocortical neurons. Neuroscience 2001;107:13-24.

Destexhe A, Rudolph M and Paré D. The high-conductance state of neocortical neurons in vivo. Nature Reviews Neurosci 2003b;4:739-51.

Fellous JM, Rudolph M, Destexhe A, Sejnowski TJ. Synaptic background noise controls the input/output characteristics of single cells in an in vitro model of in vivo activity. Neuroscience 2003;122:811-29.

Gauck V, Jaeger D. The control of rate and timing of spikes in the deep cerebellar nuclei by inhibition. J. Neurosci. 2000;20:3006-16.

Gauck V, Jaeger D. The contribution of NMDA and AMPA conductances to the control of spiking in neurons of the deep cerebellar nuclei. J. Neurosci. 2003;23:8109-18.

Haider B, Duque A, Hasenstaub AR, McCormick DA. Neocortical network activity in vivo is generated through a dynamic balance of excitation and inhibition. J Neurosci 2006;26:4535-45.

Hasenstaub A, Shu Y, Haider B, Kraushaar U, Duque A, McCormick DA. Inhibitory postsynaptic potentials carry synchronized frequency information in active cortical networks. Neuron 2005;47:423-35.

Haeusler S and Maass W. A statistical analysis of information-processing properties of lamina-specific cortical microcircuit models. Cereb Cortex 2007;17:149-62.

Hill S, Tononi G. Modeling sleep and wakefulness in the thalamocortical system. J Neurophysiol 2005;93:1671-98.

Hines ML, Carnevale NT. The NEURON simulation environment, Neural Computation 1997;9:1179-209.

Kuhn A, Aertsen A, Rotter S. Higher-order statistics of input ensembles and the response of simple model neurons. Neural Comput. 2003;15:67-101.

Kuhn A, Aertsen A, Rotter S. Neuronal integration of synaptic input in the fluctuation-driven regime. J. Neurosci. 2004;24:2345-56.

Leger J-F, Stern EA, Aertsen A, Heck D. Synaptic integration in rat frontal cortex shaped by network activity. J. Neurophysiol. 2005;93:281-93.

Lindner B, Longtin A. Comment on "Characterization of subthreshold voltage fluctuations in neuronal membranes", by M. Rudolph and A. Destexhe. Neural Comput 2006;18:1896-931.

Matsumura M, Cope T, Fetz EE. Sustained excitatory synaptic input to motor cortex neurons in awake animals revealed by intracellular recording of membrane potentials. Exp Brain Res 1988;70:463-69.

Metherate R, Ashe JH. Ionic flux contributions to neocortical slow waves and nucleus basalis-mediated activation: whole-cell recordings in vivo. J Neurosci 1993;13:5312-23. 
Monier C, Fournier J, Frégnac Y. In vitro and in vivo measures of evoked excitatory and inhibitory conductance dynamics in sensory cortices. J. Neurosci. Meth. 2008; in press.

Monier C, Chavane F, Baudot P, Graham LJ, Frégnac Y. Orientation and direction selectivity of synaptic inputs in visual cortical neurons: a diversity of combinations produces spike tuning. Neuron 2003;37:663-80.

Moreno-Bote R, Parga N. Membrane potential and response properties of populations of cortical neurons in the high conductance state. Phys Rev Lett. 2005;94:088103.

Muller E, Buesing L, Schemmel J, Meier K. Spike-frequency adapting neural ensembles: beyond mean adaptation and renewal theories. Neural Computation 2007;19:2958-3010.

Paré D, Shink E, Gaudreau H, Destexhe A, Lang EJ. Impact of spontaneous synaptic activity on the resting properties of cat neocortical neurons in vivo. J Neurophysiol 1998;79:1450-60.

Piwkowska Z, Rudolph M, Badoual M, Destexhe A, Bal T. Re-creating active states in vitro with a dynamic-clamp protocol. Neurocomputing 2005;65-66:55-60.

Pospischil M, Piwkowska Z, Rudolph M, Bal T, Destexhe A. Calculating event-triggered average synaptic conductances from the membrane potential. J Neurophysiol 2007;97:2544-52.

Press WH, Flannery BP, Teukolsky SA, Vetterling WT. Numerical Recipes. The Art of Scientific Computing. Cambridge, MA: Cambridge University Press; 1986.

Richardson MJ. Effects of synaptic conductance on the voltage distribution and firing rate of spiking neurons. Phys Rev E Stat Nonlin Soft Matter Phys 2004;69:051918.

Richardson MJ, Gerstner W. Synaptic shot noise and conductance fluctuations affect the membrane voltage with equal significance. Neural Comput. 2005;17:923-47.

Robinson HP, Kawai N. Injection of digitally synthesized synaptic conductance transients to measure the integrative properties of neurons. J Neurosci Methods 1993;49:157-65.

Roxin A, Brunel N, Hansel D. Role of delays in shaping spatiotemporal dynamics of neuronal activity in large networks. Phys Rev Lett 2005;94:238103.

Rudolph M, Destexhe A. Characterization of subthreshold voltage fluctuations in neuronal membranes. Neural Computation 2003a; 15:2577-618.

Rudolph M, Destexhe A. The discharge variability of neocortical neurons during high-conductance states. Neuroscience 2003b;119:855-73.

Rudolph M, Destexhe A. A fast-conducting, stochastic integrative mode for neocortical neurons in vivo. J. Neurosci 2003c;23:2466-76.

Rudolph M, Destexhe A. An extended analytic expression for the membrane potential distribution of conductancebased synaptic noise. Neural Computation 2005;17:2301-15.

Rudolph M, Destexhe A. On the use of analytic expressions for the voltage distribution to analyze intracellular recordings. Neural Comput. 2006; 18: 2917-22.

Rudolph M, Destexhe A. A multichannel shot noise approach to describe synaptic background activity in neurons. Eur. Physical J. 2006; B 52: 125-32.

Rudolph M, Piwkowska Z, Badoual M, Bal T, Destexhe A. A method to estimate synaptic conductances from membrane potential fluctuations. J Neurophysiol 2004;91:2884-96. 
Rudolph M, Piwkowska Z, Brette R, Destexhe A, and Bal T. Precise dynamic-clamp injection of stochastic conductances using active electrode compensation. Soc Neurosci Abstracts 2005;687.13.

Rudolph M, Pelletier J-G, Paré D, Destexhe A. Characterization of synaptic conductances and integrative properties during electrically-induced EEG-activated states in neocortical neurons in vivo. J Neurophysiol 2005;94:280521.

Rudolph M, Pospischil M, Timofeev I, Destexhe A. Inhibition determines membrane potential dynamics and controls action potential generation in awake and sleeping cat cortex. J. Neurosci. 2007;27:5280-90.

Sanchez-Vives MV, McCormick DA. Cellular and network mechanisms of rhythmic recurrent activity in neocortex. Nat Neurosci 2000;3:1027-34.

Sanchez-Vives MV, Nowak LG, McCormick DA. Cellular mechanisms of long-lasting adaptation in visual cortical neurons in vitro. J Neurosci 2000;20:4286-99.

Sharp AA, O’Neil MB, Abbott LF, Marder E. Dynamic clamp: computer-generated conductances in real neurons. J Neurophysiol 1993;69:992-5.

Shu Y, Hasenstaub A, Badoual M, Bal T, McCormick DA. Barrages of synaptic activity control the gain and sensitivity of cortical neurons. J Neurosci 2003;23:10388-10401.

Steriade M, Nunez A, Amzica F. A novel slow $(<1 \mathrm{~Hz})$ oscillation of neocortical neurons in vivo: depolarizing and hyperpolarizing components. J Neurosci 1993;13:3252-65.

Steriade M, Timofeev I, Grenier F. Natural waking and sleep states: a view from inside neocortical neurons. J Neurophysiol 2001;85:1969-85.

Suter KJ, Jaeger D. Reliable control of spike rate and spike timing by rapid input transients in cerebellar stellate cells. Neuroscience 2004;124:305-17.

Tateno T, Robinson HP. Rate coding and spike-time variability in cortical neurons with two types of threshold dynamics. J. Neurophysiol. 2006;95:2650-63.

Timofeev I, Grenier F, Steriade M. Disfacilitation and active inhibition in the neocortex during the natural sleepwake cycle: an intracellular study. Proc Natl Acad Sci USA 2001;98:1924-29.

Uhlenbeck GE and Ornstein LS, On the theory of the Brownian motion. Phys Rev 1930;36:823-41.

van Vreeswijk C, Sompolinsky H. Chaos in neuronal networks with balanced excitatory and inhibitory activity. Science 1996;274:1724-26.

Wehr M, Zador AM. Balanced inhibition underlies tuning and sharpens spike timing in auditory cortex. Nature 2003;426:442-6.

Wehr M, Zador AM. Synaptic mechanisms of forward suppression in rat auditory cortex. Neuron 2005;47:437-45.

Wilent W, Contreras D. Dynamics of excitation and inhibition underlying stimulus selectivity in rat somatosensory cortex. Nature Neurosci 2005;8:1364-70.

Wolfart J, Debay D, Le Masson G, Destexhe A, Bal T. Synaptic background activity controls spike transfer from thalamus to cortex. Nat Neurosci 2005;8:1760-67.

Woody CD, Gruen E. Characterization of electrophysiological properties of intracellularly recorded neurons in the neocortex of awake cats: a comparison of the response to injected current in spike overshoot and undershoot neurons. Brain Res 1978;158:343-57. 
Piwkowska et al., J. Neurosci. Meth. (in press, 2008)

Zou Q, Rudolph M, Roy N, Sanchez-Vives M, Contreras D, Destexhe A. Reconstructing synaptic background activity from conductance measurements in vivo. Neurocomputing 2005;65:673-78. 
Piwkowska et al., J. Neurosci. Meth. (in press, 2008)

\section{Figures}

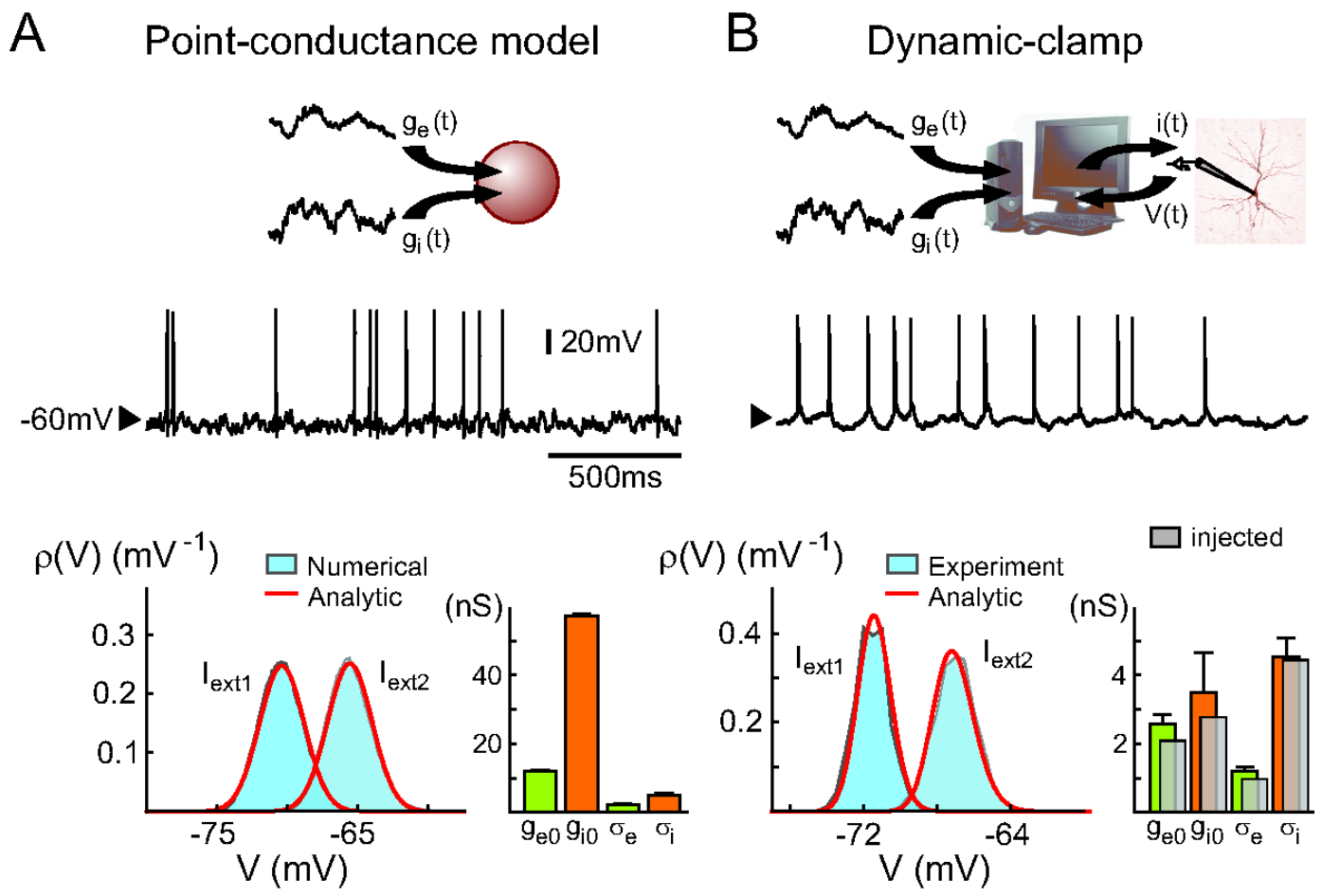

C

Natural up-state

Dynamic-clamp

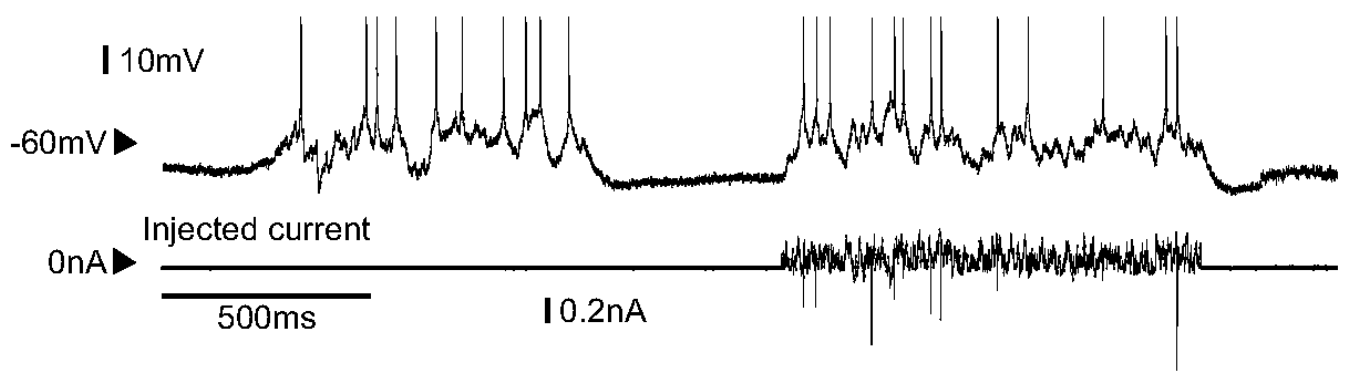

Figure 1: Conductance extraction from $\mathrm{V}_{m}$ distributions: numerical and dynamic-clamp test of the method. A. Simulation of the point-conductance model (top trace) and comparison between numerically computed $\mathrm{V}_{m}$ distributions (bottom; blue) and the analytic expression (red curves; conductance values shown in the bar graph). B. Dynamic-clamp injection of the point-conductance model in a real neuron. (Right) Conductance parameters are re-estimated (back, colored; error bars are standard deviations obtained when the same injected conductance parameters are re-estimated in different cells) from the $\mathrm{V}_{m}$ distributions and compared to the known parameters of the injected conductances (front, grey). (Left) The experimental $\mathrm{V}_{m}$ distributions are compared to the analytic distributions calculated using the re-estimated conductance parameters. C. Comparison of a spontaneous up-state (Natural up-state) with an artificial up-state recreated using conductance injection (Dynamic-clamp). Modified from Rudolph et al., 2004. 


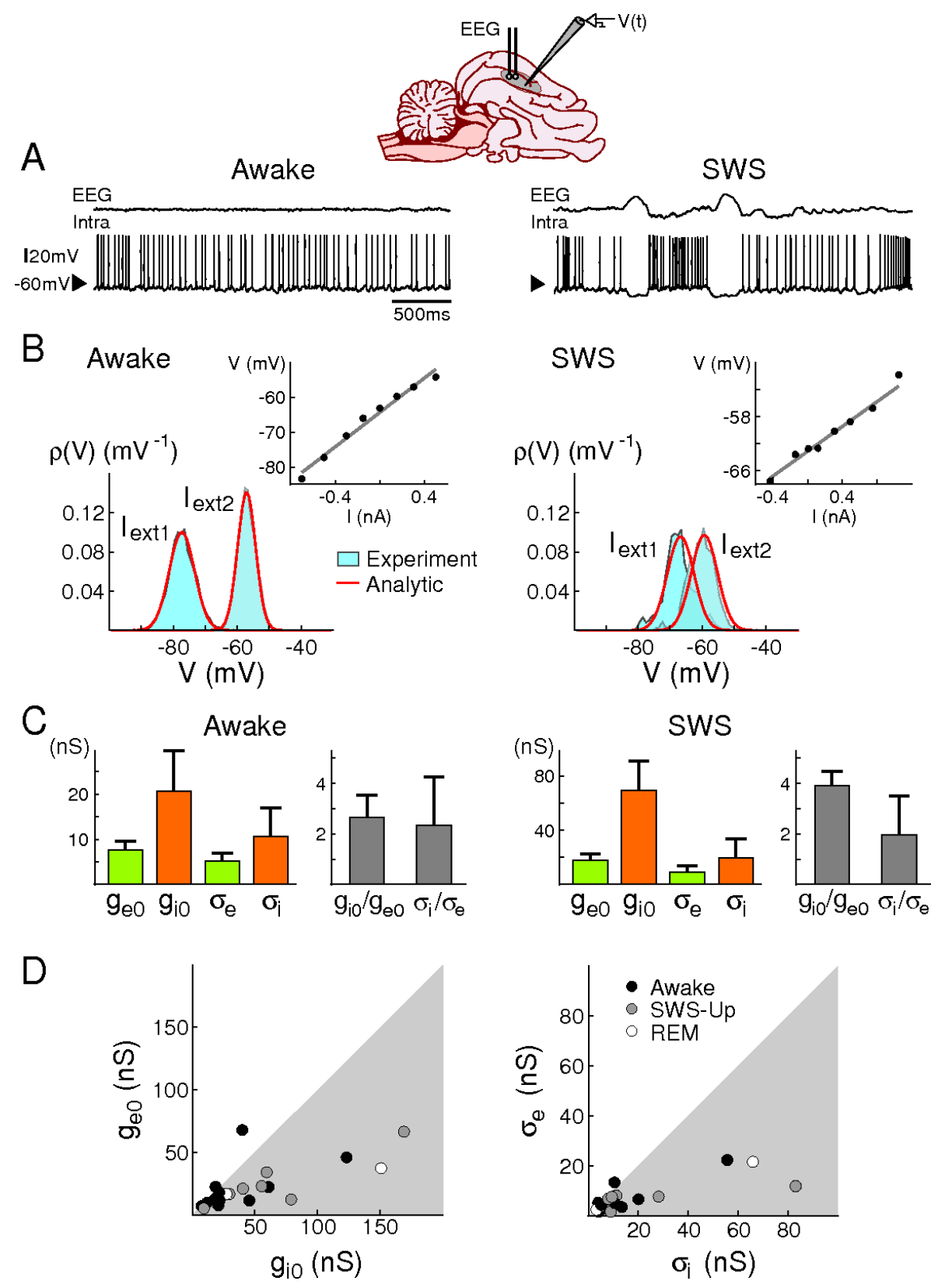

Figure 2: VmD estimation of conductances from intracellular recordings in awake and naturally sleeping cats. A. Intracellular recordings in awake and naturally sleeping (SWS) cats. Recordings were made in association cortex (area 5-7). B. Examples of $\mathrm{V}_{m}$ distributions computed during wakefulness (Awake) and slow-wave sleep up-states (SWS). The continuous lines show Gaussian fits of the experimental distributions. Insets: current-voltage relations obtained for these particular neurons. C. Conductance values estimated using the $\mathrm{VmD}$ method. Results for the means $\left(g_{e 0}, g_{i 0}\right)$ and standard deviations $\left(\sigma_{e}, \sigma_{i}\right)$ of excitatory and inhibitory conductances, respectively, as well as their ratios are shown (error bars: standard deviations obtained by repeating the analysis using different pairs of injected current levels). D. Grouped data showing the means and standard deviations of the conductances for different cells across different behavioral states (REM = Rapid Eye Movement sleep). Figure modified from Rudolph et al., 2007. 
A
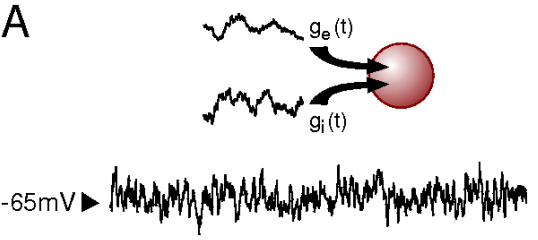

$\overline{500 \mathrm{~ms}} \quad 15 \mathrm{mV}$
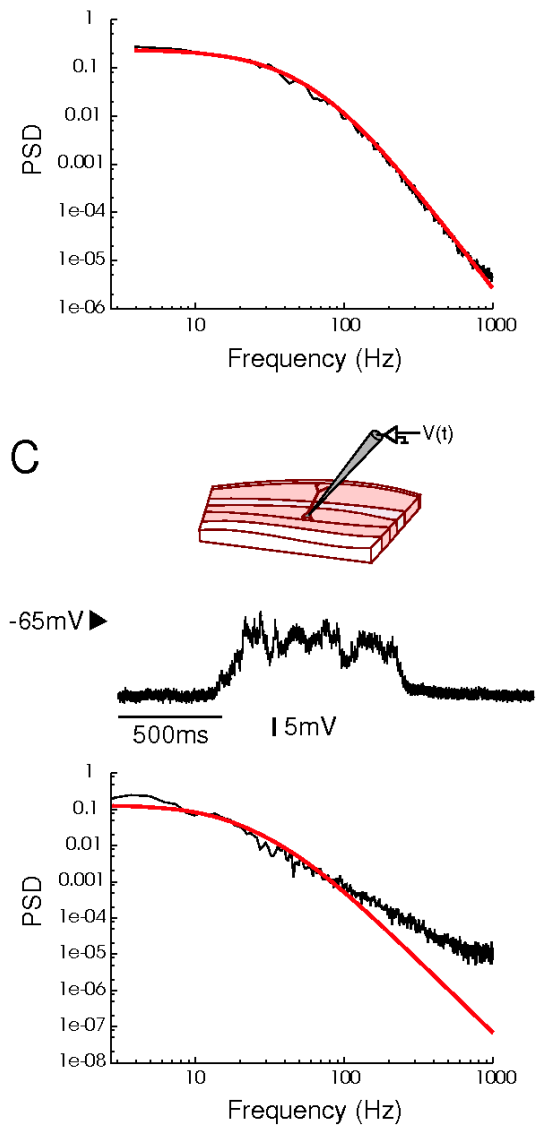

$B$
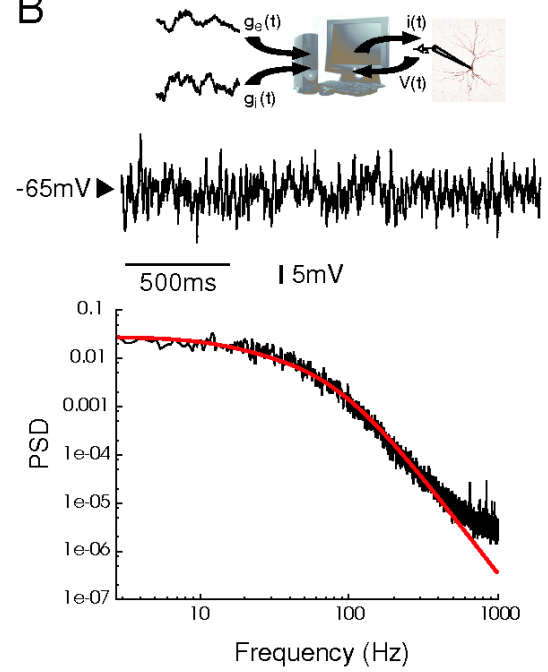

$\mathrm{D}$
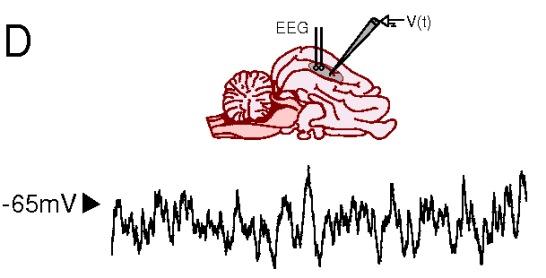

500ms $\quad 15 \mathrm{mV}$

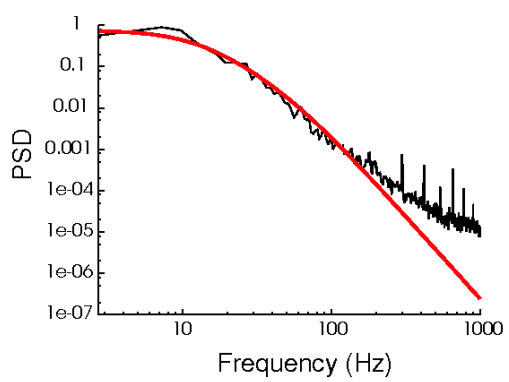

Figure 3: Fit of the synaptic time constants to the power spectrum of the membrane potential. A. Comparison between the analytic prediction (Eq. 11, red) and the PSD of the $\mathrm{V}_{m}$ for a single-compartment model (Eq. 1; black) subject to excitatory and inhibitory fluctuating conductances (Eqs. 2, 3; $\tau_{e}=3 \mathrm{~ms}$ and $\tau_{i}=$ $10 \mathrm{~ms}$ ). B. PSD of the $\mathrm{V}_{m}$ activity in a guinea-pig visual cortex neuron (black), where the same model of fluctuating conductances as in A was injected using dynamic-clamp. The red curve shows the analytic prediction using the same parameters as the injected conductances $\left(\tau_{e}=2.7 \mathrm{~ms}\right.$ and $\left.\tau_{i}=10.5 \mathrm{~ms}\right)$. C. PSD of $\mathrm{V}_{m}$ activity obtained in a ferret visual cortex neuron (black) during spontaneously occurring up-states. The PSD was computed by averaging PSDs calculated for each up-state. The red curve shows the best fit of the analytic expression with $\tau_{e}=3 \mathrm{~ms}$ and $\tau_{i}=10 \mathrm{~ms}$. D. PSD of $\mathrm{V}_{m}$ activity recorded in cat association cortex during activated states in vivo. The red curve shows the best fit obtained with $\tau_{e}=3 \mathrm{~ms}$ and $\tau_{i}=$ 10 ms. Panel A modified from Destexhe and Rudolph, 2004; Panel D modified from Rudolph et al., 2005. 


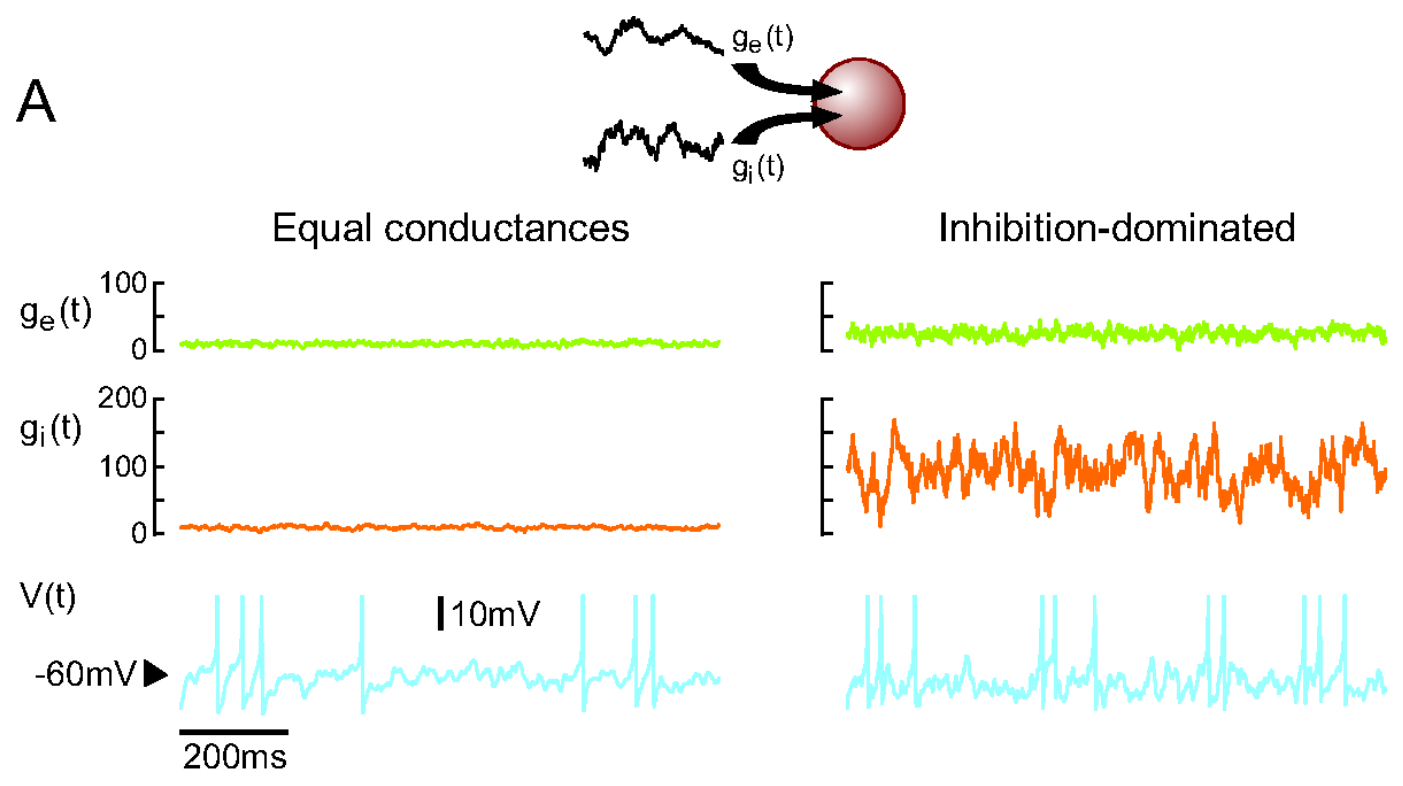

B

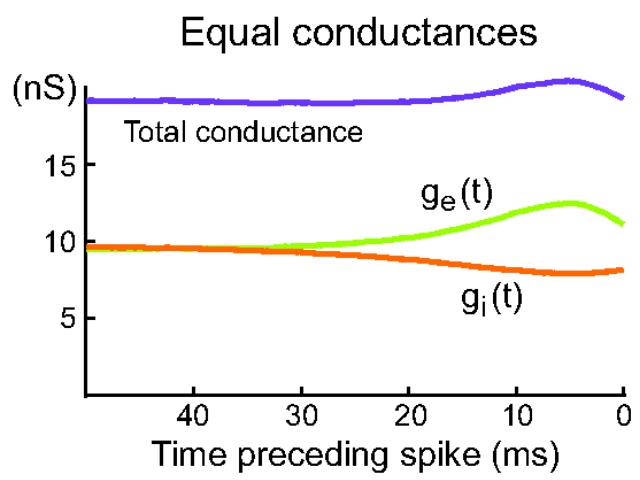

C

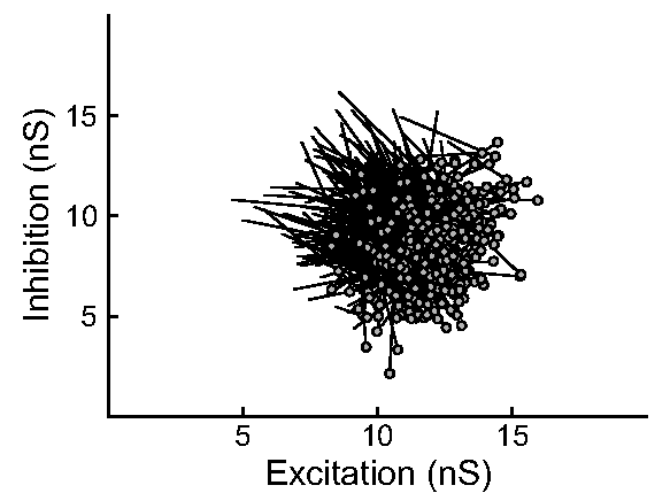

Inhibition-dominated
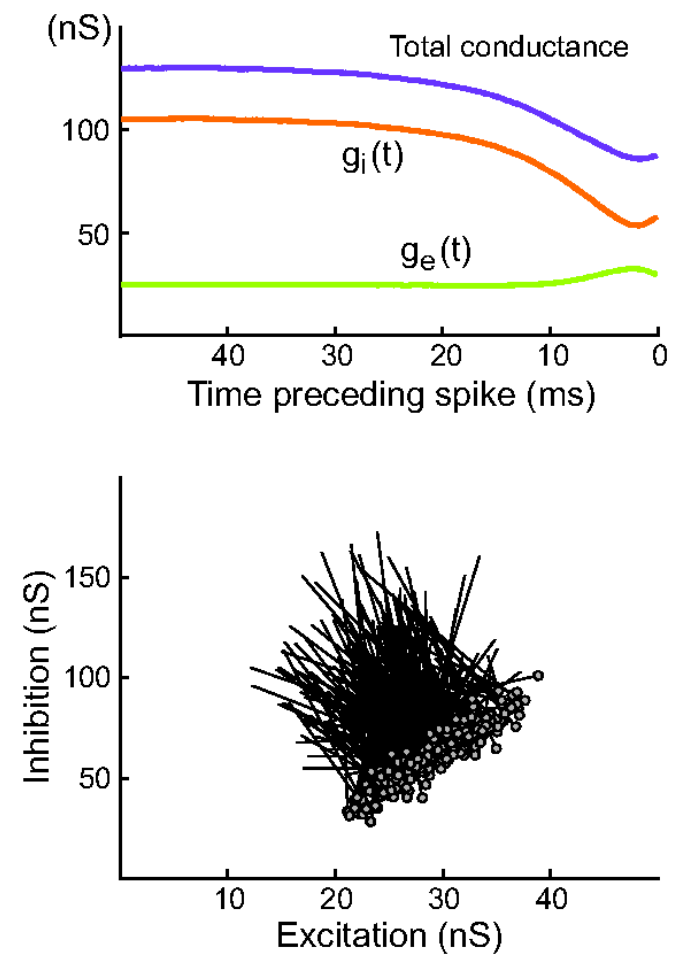

Figure 4: Comparison between equal conductances and inhibition-dominated states in a computational model. A. Equal conductance (left; $g_{e 0}=g_{i 0}=10 \mathrm{nS}, \sigma_{e}=\sigma_{i}=2.5 \mathrm{nS}$ ) and inhibition-dominated states (right; $g_{e 0}=25 \mathrm{nS}, g_{i 0}=100 \mathrm{nS}, \sigma_{e}=7 \mathrm{nS}$ and $\sigma_{i}=28 \mathrm{nS}$ ) in the point-conductance model. Excitatory and inhibitory conductances, and the membrane potential, are shown from top to bottom. Action potentials (truncated here) were described by Hodgkin-Huxley type models (Destexhe et al., 2001; Eq. 4). B. Average conductance patterns triggering spikes. Spike-triggered averages (STAs) of excitatory, inhibitory and total conductance were computed in a window of $50 \mathrm{~ms}$ before the spike. C. Vector representation showing the variation of synaptic conductances preceding each spike. The excitatory and inhibitory conductances were averaged in two windows of $30-40 \mathrm{~ms}$ and $0-10 \mathrm{~ms}$ (circle) before the spike, and a vector was drawn between the obtained values. 


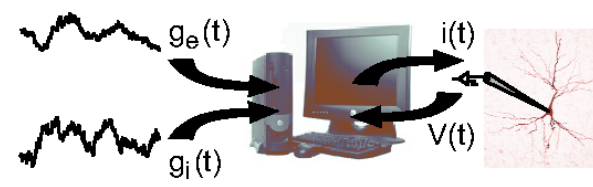

A

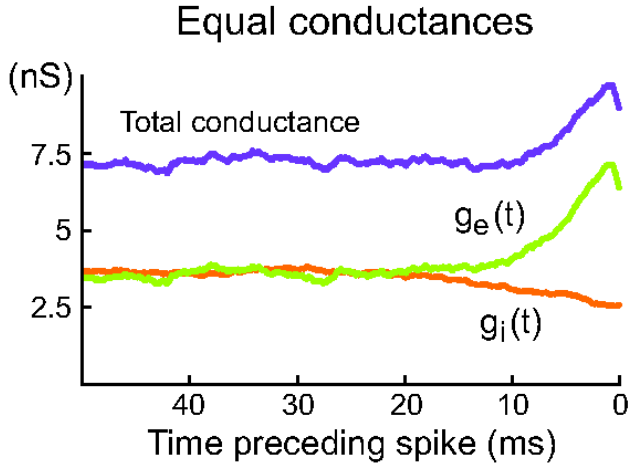

B

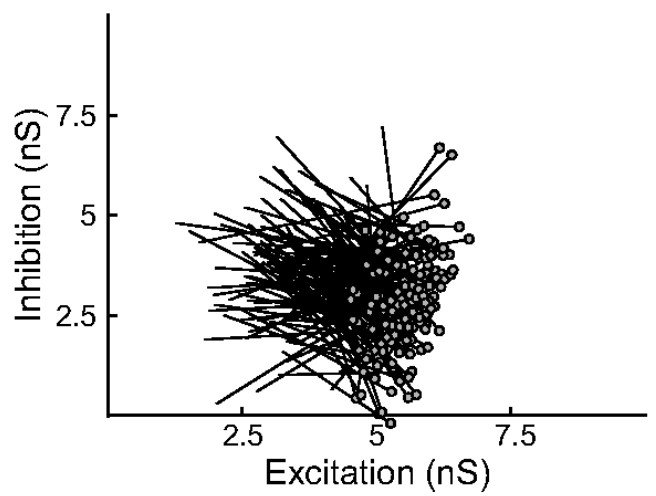

Inhibition-dominated
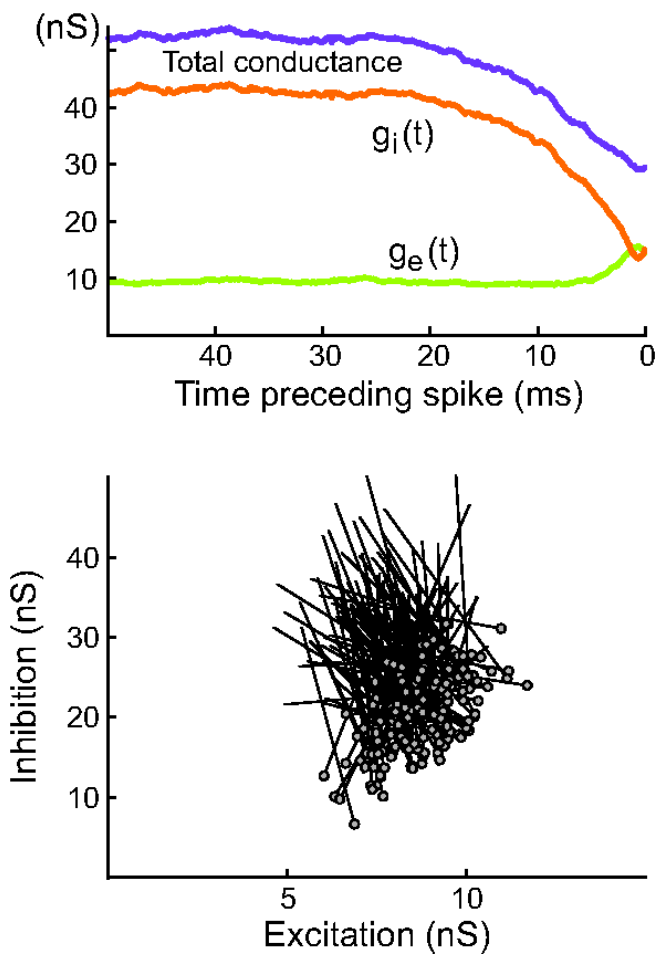

Figure 5: Average conductance patterns triggering spikes in dynamic-clamp experiments. A. Spiketriggered averages of excitatory, inhibitory and total conductance in a window of $50 \mathrm{~ms}$ before the spike in a cortical neuron subject to fluctuating conductance injection. The two states, equal conductances (left) and inhibition-dominated (right), were recreated similar to the model of Fig. 4. Conductance STAs showed qualitatively similar patterns. B. Vector representation showing the variation of synaptic conductances preceding each spike (as in Fig. 44C). 
A

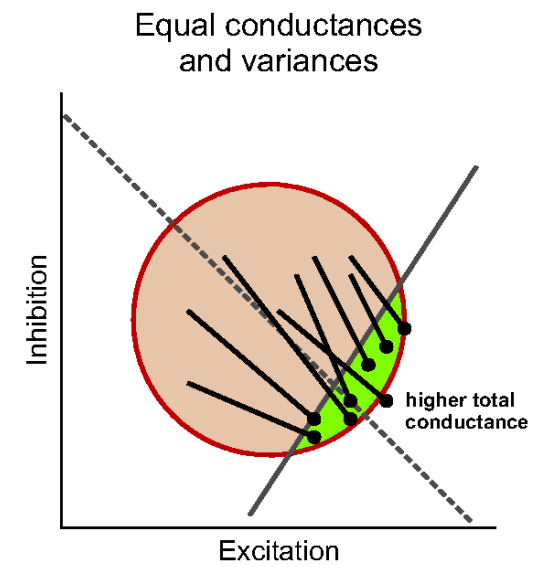

B Dominant excitatory variance
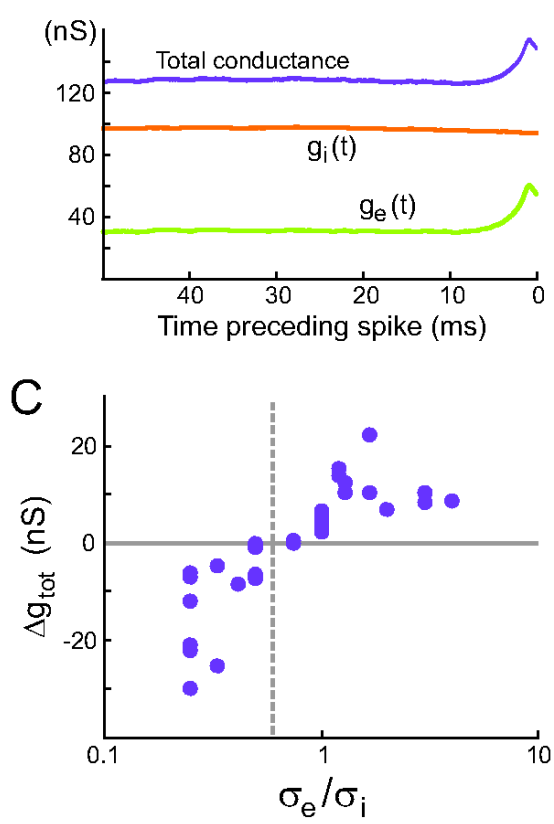

Inhibition-dominated, dominant inhibitory variances

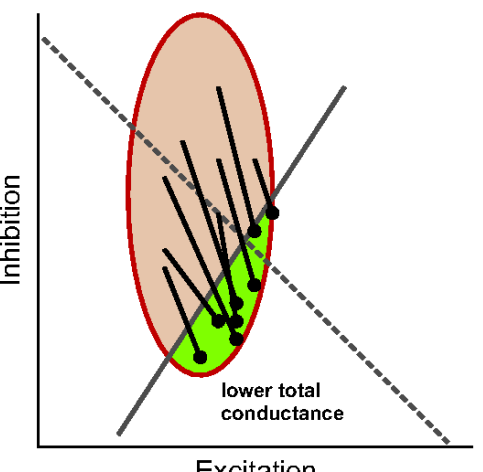

Excitation

Dominant inhibitory variance
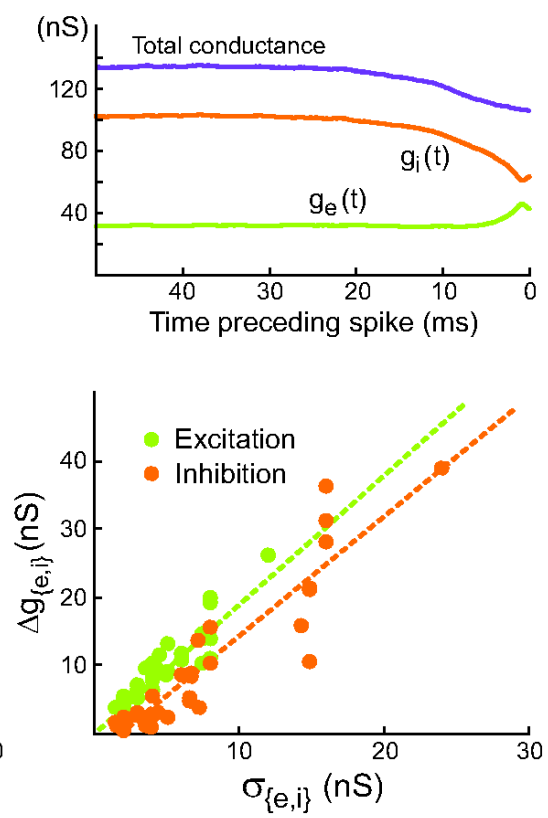

Figure 6: Geometrical interpretation of the average conductance patterns preceding spikes and test in dynamic-clamp. A. Red ellipses: isoprobable conductance configurations. Green area: conductance configurations for which the total synaptic current is positive at spike threshold. The vector representations of Figs. 45 are schematized here, and compared to the lines defined by $\left\{g_{e}\left(E_{e}-V_{t}\right)+g_{i}\left(E_{i}-V_{t}\right)+G_{l}\left(E_{L}-V_{t}\right)=0\right\}$ (solid gray line) and $\left\{g_{e}+g_{i}=g_{e}+g_{i 0}\right\}$ (dashed gray line). The angle between the two lines and the aspect ratio of the ellipse determine whether spikes are preceded, on average, by total conductance increase (left) or decrease (right) (see text for further explanations). B. Spike-triggered average conductances obtained in dynamic-clamp, illustrating that for the same average conductances, the variances determine whether spikes are preceded by total conductance increase (left) or decrease (right). C. Geometrical prediction tested in dynamic-clamp (left): grouped data showing total conductance change preceding spikes as a function of the ratio $\sigma_{e} / \sigma_{i}$. The dashed line $\left(\sigma_{e} / \sigma_{i}=0.6\right)$ visualizes the predicted value separating total conductance increase cases from total conductance decrease cases. In addition (right), dynamic-clamp data indicates that the amplitude of change of each of the conductances before a spike is linearly correlated with the standard deviation parameter used for this conductance. 

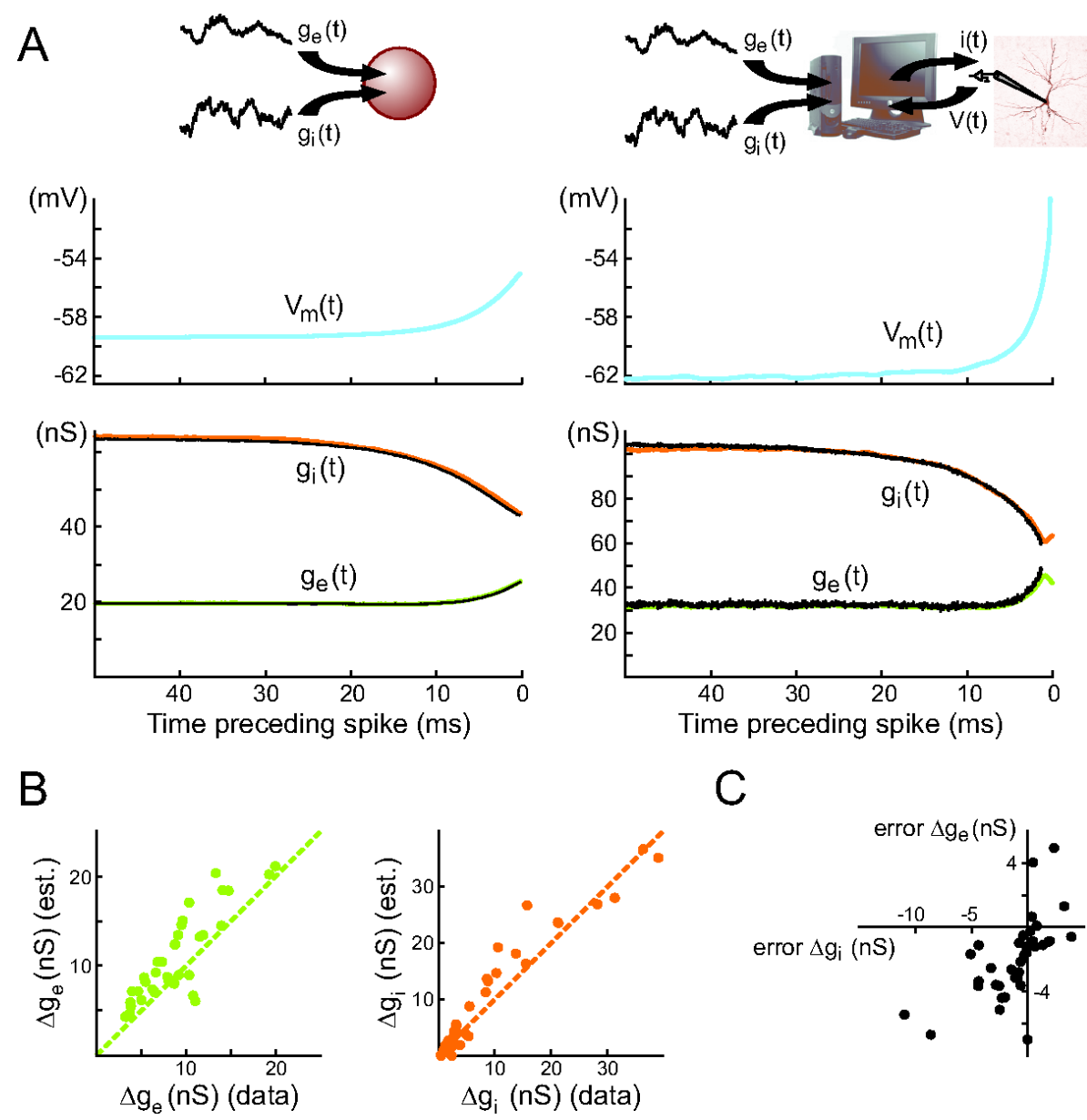

C
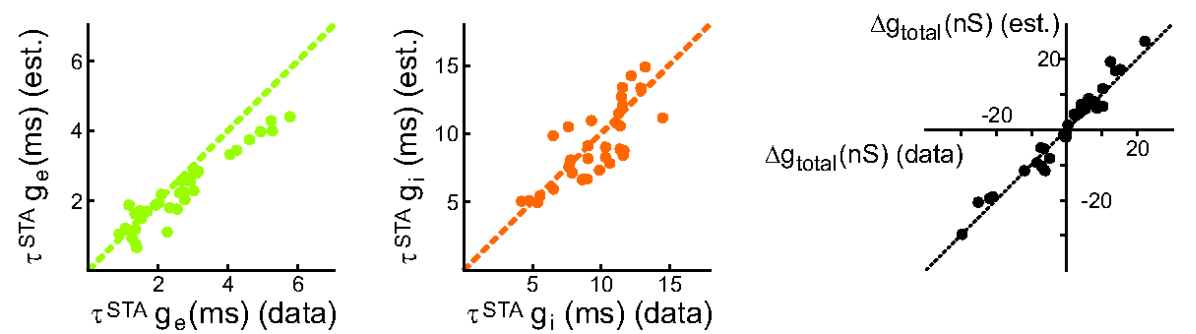

Figure 7: Spike-triggered conductance extraction from intracellular recordings of the $V_{m}$ and test in dynamic-clamp. A. Left: spike-triggered average (STA) of the $\mathrm{V}_{m}$ in an integrate-and-fire extension of the point-conductance model (top trace). The numerically obtained conductance STAs (orange, green) are compared to the conductance STAs extracted from the $\mathrm{V}_{m}$ (black) (bottom trace). Right: test of the STA method using dynamic-clamp. The STA of the $\mathrm{V}_{m}$ is obtained following injection of fluctuating conductances (top trace). The measured conductance STAs (orange, green) are compared to the conductance STAs extracted from the $\mathrm{V}_{m}$ (black) (bottom trace). B. Grouped data comparing conductance STAs extracted using the method with the conductance STAs measured following dynamic-clamp injection: amplitude of conductance change preceding the spike (top graphs) and time constant of this change (bottom graphs), for both excitation and inhibition. C. Top: correlation between errors for excitation and inhibition on the absolute value of conductance variation. Bottom: total conductance change preceding spikes; comparison between extracted and measured STAs. Dashed lines: $Y=X$. 

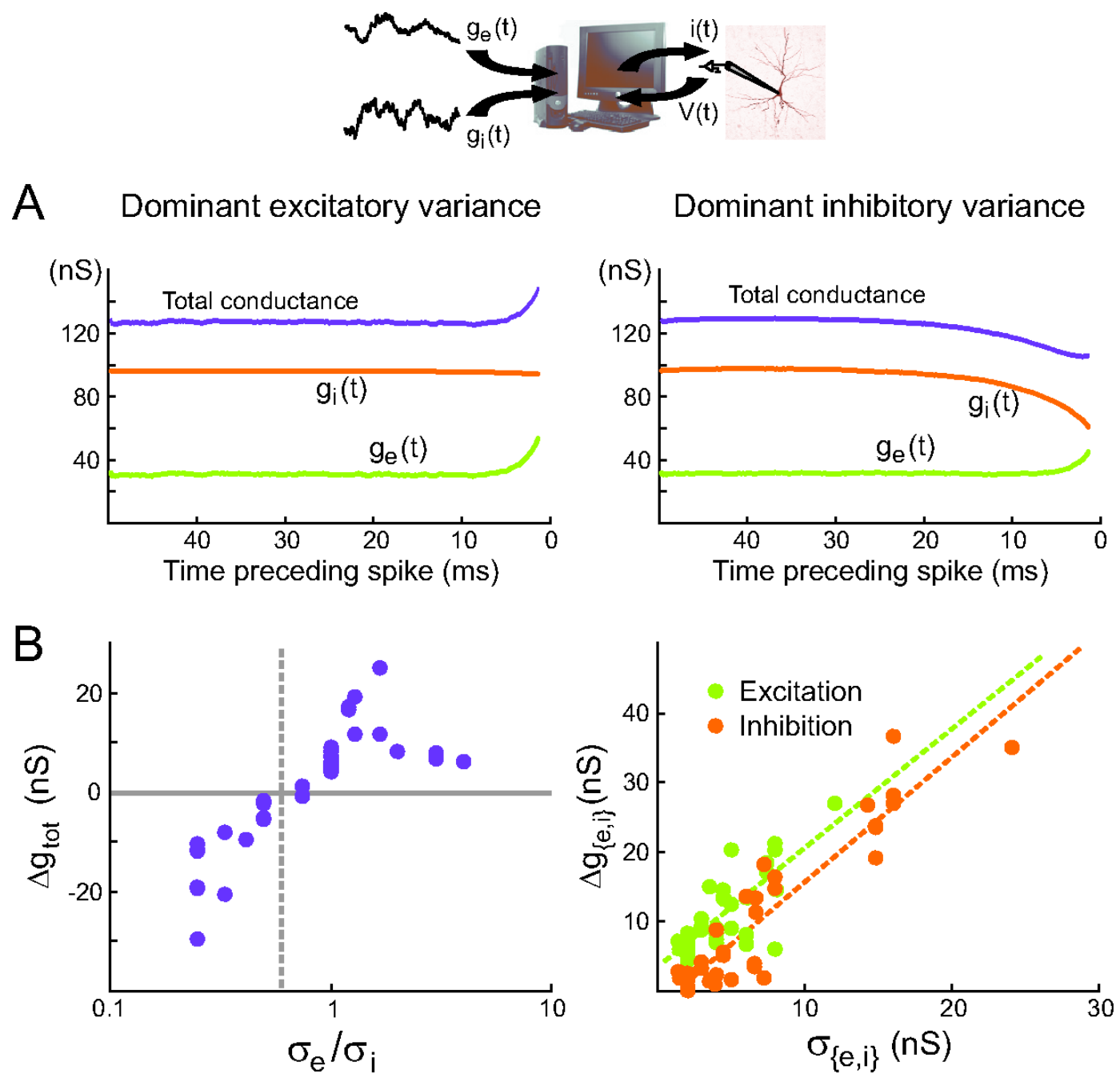

Figure 8: Analysis of the average conductance patterns preceding spikes, same analysis as Fig. 6B-C, but using conductance STAs extracted from the $\mathrm{V}_{m}$, instead of the measured ones. A. Example conductance STAs extracted from the $\mathrm{V}_{m}$ STAs of the same cell and the same conductance injections as Fig. 6B. B. Left: test of the geometrical prediction (dashed line) using conductance STAs extracted from the $\mathrm{V}_{m}$, and showing total conductance change preceding spikes as a function of the ratio $\sigma_{e} / \sigma_{i}$ (as in Fig. 6C, left). Right: correlation between the amplitude of change of each conductance preceding a spike, as extracted from the $\mathrm{V}_{m}$, and the standard deviation parameter for this conductance (compare to Fig. 6C, right). 

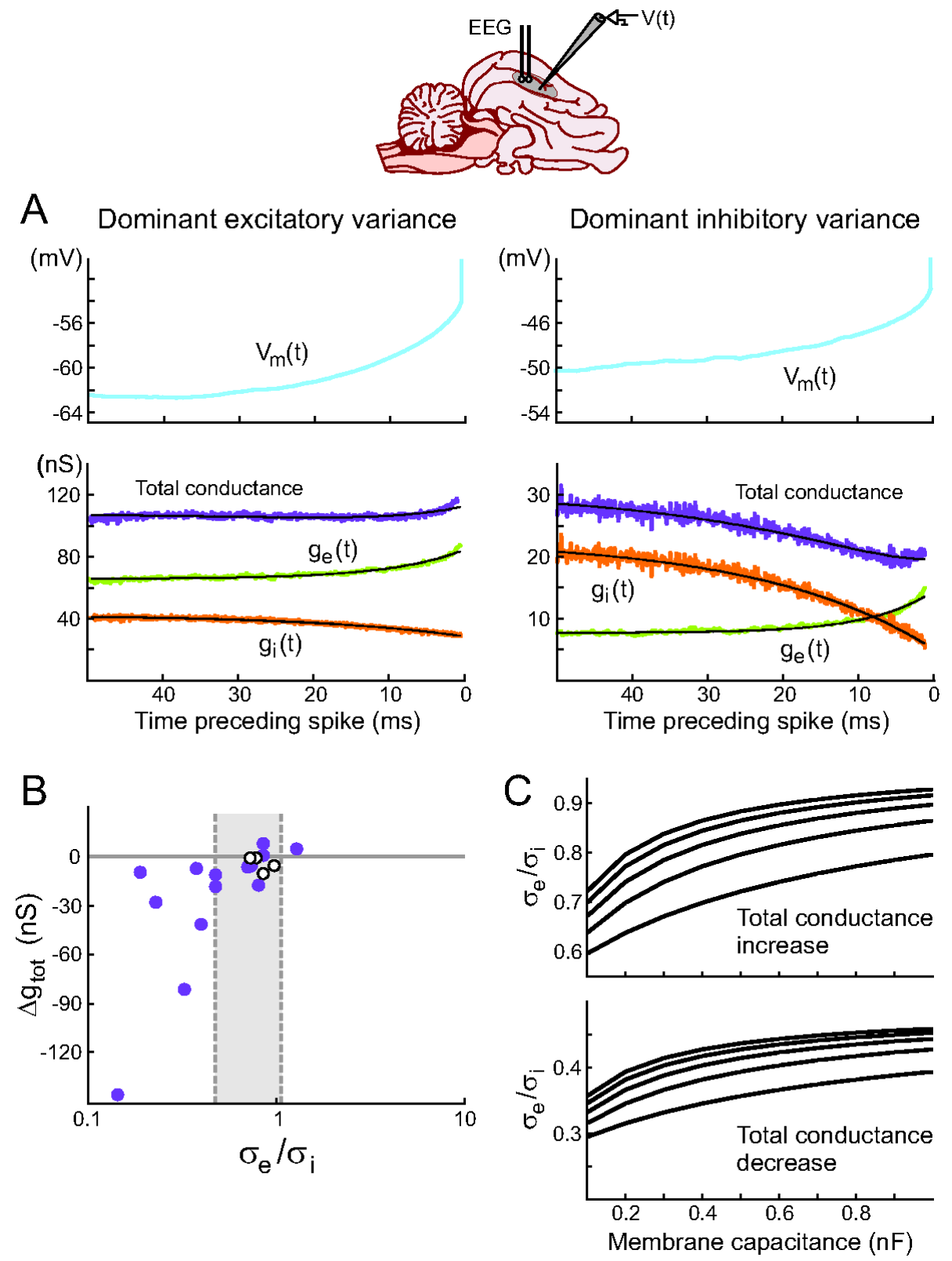

Figure 9: Spike-triggered conductance analysis in vivo. A. STA conductance analysis from intracellular recordings in awake and sleeping cats. Two example cells are shown during wakefulness, and for each, the $\mathrm{V}_{m}$ STA (top) and the extracted conductance STAs (bottom) are shown. In the first cell (left), the total conductance increases before the spike. In the second example cell (right), the total conductance decreases before the spike (black traces are exponential fits to the extracted STAs). B. Total conductance change preceding spikes as a function of the ratio $\sigma_{e} / \sigma_{i}$. Given the cell-to-cell variability of observed spike thresholds, each cell has a different predicted ratio separating total conductance increase cases from total conductance decrease cases. The two dashed lines $\left(\sigma_{e} / \sigma_{i}=0.48\right.$ and $\left.\sigma_{e} / \sigma_{i}=1.07\right)$ visualize the two extreme predicted ratios. Cells in white are the ones not conforming to the prediction. C. Dependency of the ratio $\sigma_{e} / \sigma_{i}$ estimated by the $\mathrm{VmD}$ method on the value of the membrane capacitance $C$. Two sets of realistic $\mathrm{V}_{m}$ distribution parameters were used as input for the estimation, one leading to $\sigma_{e} / \sigma_{i}>0.6$ (left), another leading to $\sigma_{e} / \sigma_{i}<0.6$ (right). For each set, the total input resistance was varied from $10 \mathrm{M} \Omega$ (bottom curves) to $50 \mathrm{M} \Omega$ (top curves), in steps of $10 \mathrm{M} \Omega$. Panel A modified from Rudolph et al., 2007. 